\title{
Crosslinking Trends in Multicomponent Hydrogels for Biomedical Applications
}

\author{
Jagan Mohan Dodda,* Mina Ghafouri Azar, and Rotimi Sadiku
}

Multicomponent-based hydrogels are well established candidates for biomedical applications. However, certain aspects of multicomponent systems, e.g., crosslinking, structural binding, network formation, proteins/drug incorporation, etc., are challenging aspects to modern biomedical research. The types of crosslinking and network formation are crucial for the effective combination of multiple component systems. The creation of a complex system in the overall structure and the crosslinking efficiency of different polymeric chains in an organized fashion are crucially important, especially when the materials are for biomedical applications. Therefore, the engineering of hydrogel has to be, succinctly understood, carefully formulated, and expertly designed. The different crosslinking methods in use, hydrogen bonding, electrostatic interaction, coordination bonding, and self-assembly. The formations of double, triple, and multiple networks, are well established. A systematic study of the crosslinking mechanisms in multicomponent systems, in terms of the crosslinking types, network formation, intramolecular bonds between different structural units, and their potentials for biomedical applications, is lacking and therefore, these aspects require investigations. To this end, the present review, focuses on the recent advances in areas of the physical, chemical, and enzymatic crosslinking methods that are often, employed for the designing of multicomponent hydrogels.

\section{Introduction}

Hydrogels are highly hydrated, 3D, cross-linked polymer networks that are increasingly, finding usage in biomedical applications, such as drug delivery, injectates, antimicrobials, biosensors, medical devices, tissue engineering, and bio-actuators. An adult human body contains $\approx 60 \%$ of water and the properties of

\section{J. M. Dodda, M. G. Aza}

New Technologies-Research Centre (NTC)

University of West Bohemia

Univerzitní 8, Pilsen 301 00, Czech Republic

E-mail: jagan@ntc.zcu.cz

R. Sadiku

Institute of NanoEngineering Research (INER) and Department of

Chemical, Metallurgical and Materials Engineering

Tshwane University of Technology

Staatsartillerie Rd, Pretoria West Campus, Pretoria 0183, Republic of South Africa

The ORCID identification number(s) for the author(s) of this article can be found under https://doi.org/10.1002/mabi.202 100232

DOI: 10.1002/mabi.202100232 hydrogels, such as swelling, stretching, softness, insolubility, etc., are considered as beneficial for current biomedical research. Hydrogel came into light with the establishment of the first synthetic hydrogels on crosslinked hydroxyethylmethacrylate (HEMA) by Wichterle and $\operatorname{Lim}^{[1]}$ in 1954. In 1980, Lim and Sun demonstrated the successful application of calcium alginate microcapsules for cell encapsulation ${ }^{[2]}$ and later on, Gin and co-workers utilized them for rat implantation. ${ }^{[3]}$ The presence of hydrophilic functionalities (such as $-\mathrm{OH},-\mathrm{NH}_{2},-\mathrm{COOH}$, and $-\mathrm{SO}_{3} \mathrm{H}$ ) in the polymer chain and crosslink networks, governs the water absorption capacity, insolubility and mechanical properties of hydrogels. ${ }^{[4-8]}$ Due to their ecofriendly characteristics of preparation (by natural polymer, such as starch, chitosan, agarose, etc.) and their excellent biocompatibility characters, allow these soft materials to be engineered in order to mimic the native tissues and be integrated with other materials (e.g., nanomaterials, ceramics, etc.) in order to produce composites suitable for organs of the human body. ${ }^{[9-12]}$ Over the years, the progress in hydrogel synthesis has undergone many transitions and currently, significant attention is focused on hydrogels in order to derive ecofriendly hydrogels by the use of naturally-occurring polysaccharides or biocompatible polymers, considering the key issues that are, related to biodegradability and environmental protection.

Conventionally, hydrogels are prepared in two ways: a) via a 3D polymerization or b) by direct crosslinking of hydrophilic polymers (Figure 1b). In the 3D polymerization route, the hydrophilic monomers (e.g., acrylates and vinyl monomers) are polymerized with multifunctional cross-linking agents in order to form the hydrogels of the required structures. However, the gels obtained may contain a substantial amount of residual monomers and hence, they need to be purified thoroughly in order to remove any unreacted/residual monomers. ${ }^{[13]}$ The purification is usually performed by extraction into excess water and can take-up several weeks to be completed. ${ }^{[14,15]}$ Many researchers have reported the use of additional procedures in order to obtain the maximum possible monomer conversion via the $3 \mathrm{D}$ polymerization route and consequently, employ the post-polymerization curing techniques by thermal or irradiation of resultant products. ${ }^{[16,17]}$ Alternately, non-toxic monomers/macromer (e.g., polyethylene glycol 

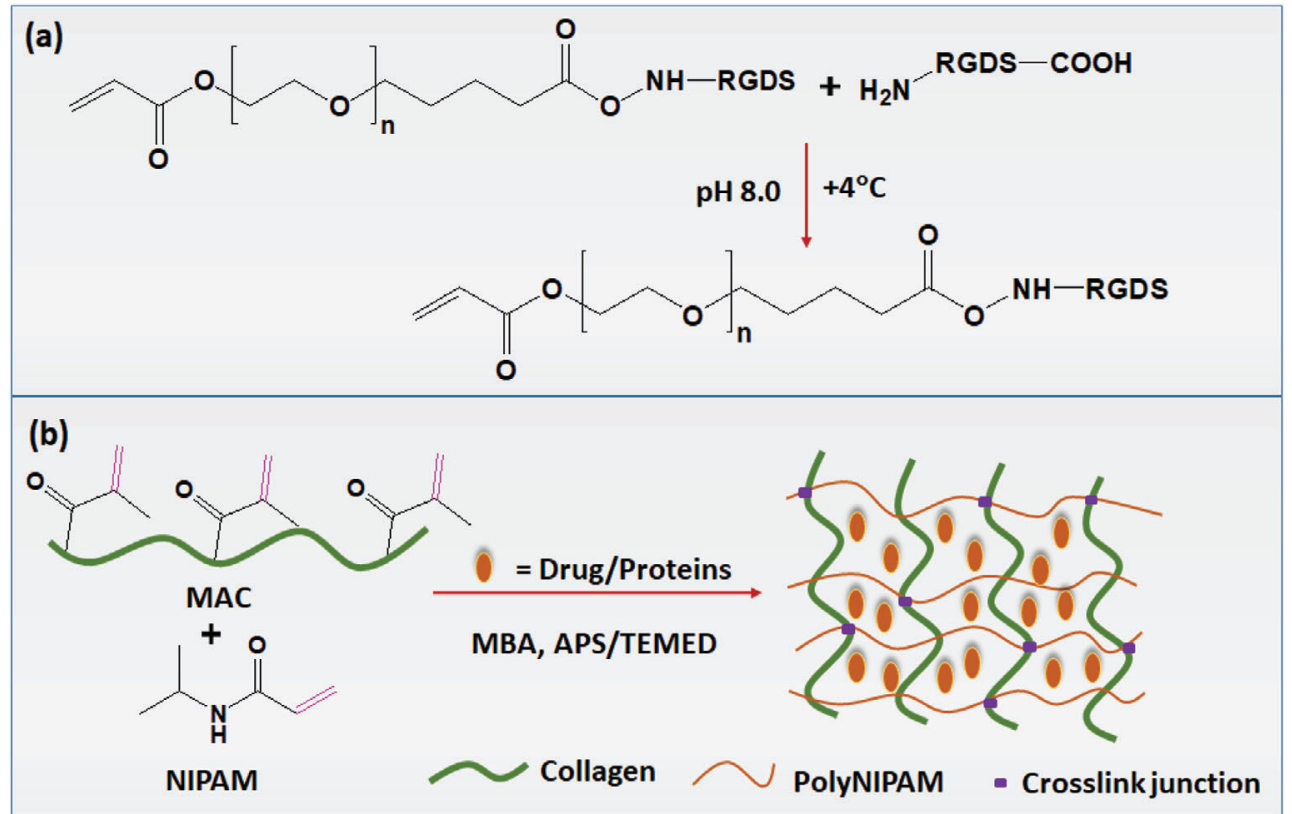

Figure 1. a) Synthesis of bioactive acryl-PEG-peptide. Formation of cell adhesive PEG-RGDS. The amine-reactive PEG derivate reacts with peptide (RGDS) at $\mathrm{N}$-terminus, forming PEG chain extension with the peptide. Subsequently, the peptide is grafted into the hydrogel matrix during the crosslinking process and b) synthesis of injectable MAC-NIPAM hydrogel: scheme demonstrating the synthesis of injectable hydrogels under physiological conditions by free radical co-polymerization of MAC and NIPAM in presence of MBA (crosslinking agent), APS, and TEMED (redox initiators).

dimethacrylate) or water soluble polymers (e.g., poly(acrylic acid), poly(vinyl alcohol), polyethylene glycol) or some polysaccharide can be utilized for the hydrogel preparation, without the need for any purification. For example, poly(ethylene glycol) diacrylate (PEGDA)-based hydrogels have been extensively used in regenerative engineering applications due to its ability to encapsulate cells, resist protein absorption, control bioactive ligands, and superior mechanical properties. In order to render cell adhesion, peptide ligands, for example, RGDS (arginine-glycine-aspartic acid-serine) have been incorporated into the PEGDA hydrogel by using an amine reacted PEG derivative. The amine-reactive PEG derivative, that is, acrylate-PEG-succinimidyl valerate (an $N$-hydroxysuccinimide ester group) reacts with RGDS by amine substitution, to form acryl-PEG-RGDS hydrogel (Figure 1a). The effective crosslinking act between RGDS and PEGDA, created strong bonding between the RGDS and the internal receptors on the cell incorporated in PEGDA hydrogels, resulting in good cell adhesion. ${ }^{[18]}$

For a direct crosslinking, the polymerization is usually initiated by free-radical generating-compounds, for example, benzoyl peroxide, 2, 2-azo-isobutyronitrile, and ammonium peroxodisulphate (APS) or by means of ultraviolet, gamma, or electron beam radiation. ${ }^{[19]}$ Free radical polymerization is the most preferred technique for preparing hydrogel, since the polymer is shaped by sequential addition of free radical groups, which act as templates for the structural designing of hydrogel. For example, injectable collagen hydrogels have been successfully produced under physiological conditions by the free radical copolymerization of methacrylated type-I collagen (MAC) and $\mathrm{N}$-isopropyl acrylamide (NIPAM), while APS and N, N,N,Ntetramethylethylenediamine (TEMED) were used as redox ini- tiators and N,N-methylenebis(acrylamide) (MBA) as crosslinking agent (Figure 1b). The hydrogels showed sustained release of bovine serum albumin (BSA) and vitamin E succinate over 2 weeks, indicating its suitability for the delivery of drug molecules and proteins. ${ }^{[20]}$

A wide range of polymers of natural and synthetic origins are used for the fabrication of hydrogels, however, multicomponent formulations with sufficient engineerability that has wide applicability and proven specific applications are required for current biomedical needs (Figure 2). Natural polymers mainly include polysaccharides and proteins obtained from plant and animal sources. In 1989, Yannas and co-workers, ${ }^{[21]}$ prepared a hydrogel by using collagen and shark cartilage for use in wound dressings. Later, these biocompatible hydrogels were extensively used in tissue engineering for the development of matrices for cell growth and reframing organs of various tissues. ${ }^{[22-25]}$ For the past few decades, natural polymers are gradually being overtaken by synthetic polymers, which possess high water absorption, high gel strength, and longer sustainability. ${ }^{[26,27]}$ These polymers are chemically stronger when compared to their natural polymer counterparts.

Synthetic polymers with chemical linkages have controlled structures, process flexibility, high chemical purity, and resistance to hydrolytic and oxidative degradation with no immunological restrictions. These polymers have the ability to modulate their mechanical properties and biological inertness that needed to be tailored in order to apply them in the medical fields, such as suture materials, medical implants, dialysis tubes, fracture fixation devices, etc. ${ }^{[28-31]}$ Most synthetic hydrogels are often prepared from hydrophilic polymers, such as polyhydroxyethylmethacrylate, ${ }^{[32-34]}$ derivatives of polyacry- 

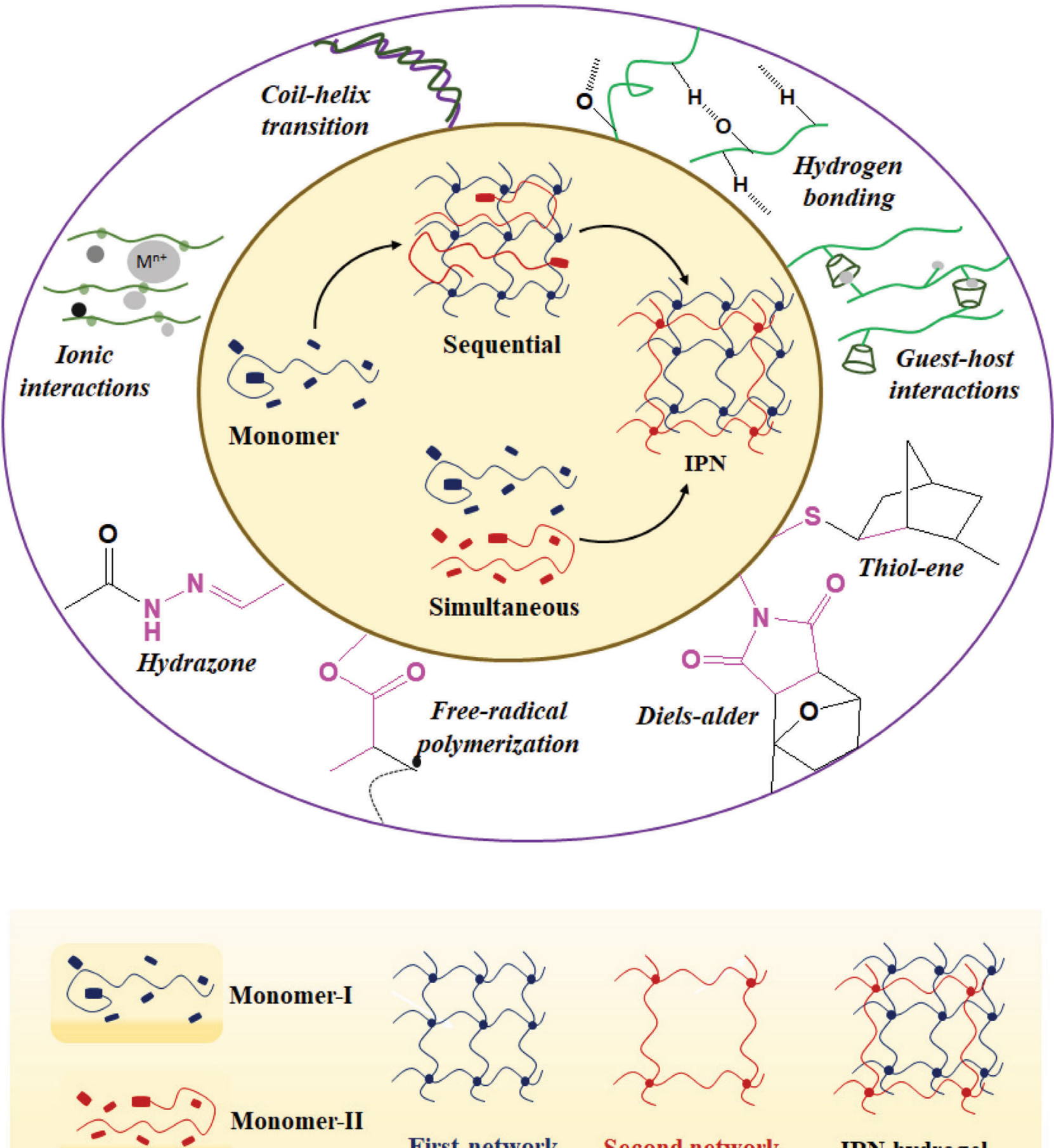

\section{Monomer-II}

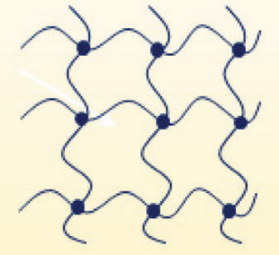

First network

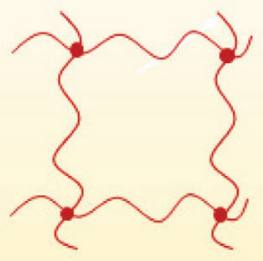

Second network

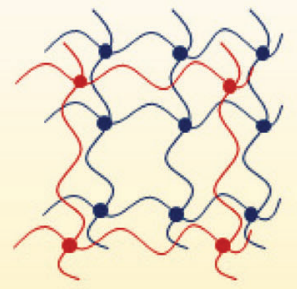

IPN hydrogel

Figure 2. Preparation of IPN hydrogels. The IPN synthesis method can be divided into sequential (swelling of first network in a secondary monomer) and simultaneous (crosslinking of first and second networks). The type of monomer/polymer and the crosslinking strategy used for each network, effects the interactions among networks within IPN hydrogel. Different approaches of physical crosslink (top right, in green) include ionic crosslinking (e.g., chitosan, alginate, guar gum, hyaluronic acid, etc.), helix-coil transition (e.g., carrageenan, agarose, gelatin), hydrogen bonding (e.g., cellulose, starch, dextran), and guest-host interactions among biopolymers. Crosslinking agents, such as glutaraldehyde, genipin, or carbodiimide are also used for direct crosslinking of polymers. The biopolymers can also be chemically-modified with functional groups for covalent crosslinking (bottom in pink), such as, hydrazone, Diels-Alder, disulfide linkage, and free radical polymerization (e.g., methacrylates). 
lamide (PAM), ${ }^{[35,36]}$ poly(vinyl alcohol) (PVA), ${ }^{[37,38]}$ polyacrylic acid, ${ }^{[39,40]}$ poly(ethylene glycol) (PEG), ${ }^{[41,42]}$ etc. Lately, synthetic polymer was combined with natural polymers to enhance the biological and mechanical properties of resulting hydrogels, such as chitosan/PEG, agarose-PAM, agarose/polydopamine hydrogel, chitosan/PAM hydrogel, and chitosan-PVA. ${ }^{[3-48]}$

In recent years, the medical field has attracted projects towards the formulations of innovative hybrid-hydrogels with a synergistic combination of natural and biocompatible synthetic polymers, with the aim to protect the environment from harmful chemicals and reduce production-costs, which indirectly affect human life. Since hydrogels are used in a range of biomedical applications, such as: tissue engineering, ${ }^{[49]}$ wound healing, ${ }^{[50,51]}$ drug carriers, ${ }^{[52]}$ cancer treatments, ${ }^{[53,54]}$ etc., the gels produced, usually come directly into contact with tissues, blood vessels, internal organs, and as such, can affect the human body in many ways. Often, it can be difficult to employ these hydrogels directly in the human body, especially when the formation, properties and the degradation protocols of these materials have not been properly understood. If these issues are not addressed appropriately, it may result in serious side-effects in future. In order to overcome these drawbacks, modern health care instruments (such as electronic sensors, actuators, ${ }^{[55]}$ injectable devices, ${ }^{[56]}$ wearable electronics, ${ }^{[57]}$ artificial muscles, ${ }^{[58]}$ and electronic skin ${ }^{[59]}$ ) have implemented the use of multicomponent hydrogels to investigate the internal behavior of tissues/cells from the external devices.

Conductive hydrogel is composed of a unique combination of a conductive network and nonconductive hydrogel matrix that can be tuned for use in bioelectronics devices. The fabrication of stretchable conducting hydrogel with high conductivity and robust mechanical properties is still considered as a great challenge due to the relatively weak interactions among conductive material and polymer networks in an aqueous environment. Although there are many studies on the physically-crosslinked conductive hydrogel with different interaction structures, ${ }^{[60,61]}$ the crosslinked hydrogels have relatively large elongation, resulting in viscoelastic deformation during the electromechanical performance. Hence, many research groups are currently focusing on developing an elastic conductive hydrogel, for example, hybrid crosslinking and double networking hydrogels in order to enhance the mechanical properties of the hydrogels. ${ }^{[62-64]}$ The properties of the conducting hydrogel vary, depending on the type of conductive filler, crosslinking, and the components involved in structuring a hydrogel matrix. ${ }^{[65]}$ For practical application, the conductive gels need to be biocompatible and mechanically strong with good flexibility in order to resist high stressstrains created by the movement of internal tissues/cells of humans.

In the present review, the most recent (in the last 2 years) progress in crosslinking techniques, employed for the fabrication of multicomponent-based hydrogels for biomedical needs are, summarized. Crosslinking strategies, such as sequential, simultaneous, self-crosslinking, and network formation by physical/chemical bonding in multicomponent systems are discussed. The utilization of these multicomponent hydrogels in the biomedical field and the procedures employed to improve the mechanical stability and biocompatibility of hydrogels, are other major aspects of the current review.

\section{Crosslinking Strategies}

The formation of hydrogel is a complex process, which needs a better understanding of the basic chemistry among the polymeric constituents and their crosslinking networks. The physical properties of hydrogels are, significantly altered by the addition of crosslinks between polymer chains. Moreover, other characteristic features, such as polymer concentration, molecular weight, chain rigidity, and crosslink density can be altered in order to achieve hydrogel with the desired properties. ${ }^{[66-70]}$

Hydrogels have several unique characteristics that are comparable with extracellular matrix (ECM) that conditions cell geometry and thus, they have the potential for cell proliferation and migration during tissue regeneration and wound healing. The stabilization of encapsulated and transplanted cells, require the controlled release of drugs and they exhibit minimal mechanical irritation to the surrounding tissue. Hydrogels have a high water absorption capability, high degree of flexibility, and they are a stimuli-responsive, soft structure, network structure and their close resemblance to living tissues makes them an ideal candidate for implantation, as they introduce low levels of foreign elements into the body and allow high diffusivity of biomolecules. The biocompatibility of hydrogels (derived from naturally occurring polymers, e.g., agarose, chitosan, hyaluronan, gelatin, alginate, etc.) is well reported and the latest technologies (e.g., optical projection lithography, bioprinting, microfluidics, and biopatterning) provide the methods to design scaffold with intricate structures for various biomedical applications. ${ }^{[71-74]}$ However, the construction of functional tissues, still remains a challenge, since it relies on many parameters, which include polymer structure, cell-biomaterial interactions, encapsulated materials, etc. Thus, the designed material must offer properties (i.e., good mechanical strength, stability, and biocompatibility) that lead to cellular function in a natural manner. The hydrogel offers distinct advantages when they are utilized for developing scaffolds for tissue engineering, their physico-chemical properties, mechanical properties, cell encapsulation, drug delivery, etc., can easily be modified.

Multicomponent reactions (MCR) have received immense attention in polysaccharide chemistry. This is due to their increased usage in the biomedical sector, which not only results in creating green technology, but also produces the diversity of their products by combining polysaccharides with biocompatible polymers of different chemical structures. The multicomponent systems provide an opportunity to exploit the inherent advantages of the individual component as well as the collective effect, as a consequence of their mutual interactions. MCR reactions usually include, at least, three components reaction containing amide, carbonyl, hydroxyl, or carboxylic acid groups in the polymer chain. These multicomponent reactions are useful for designing more complex structures, for enhanced modularity and offering control of the self-assembly. ${ }^{[75-77]}$ Despite these developments, it is still a challenge to predict the properties of multicomponent assembly from predesigned molecular architecture and therefore, requires an in-depth investigation in order to produce ecofriendly multicomponent species for biomedical applications.

Presently, there is significant interest to design hydrogels of multicomponent polymers, based on sequential or simultaneous crosslinking to formulate interpenetrating polymer networks (IPNs), as shown in Figure 2. These networks are formed at 


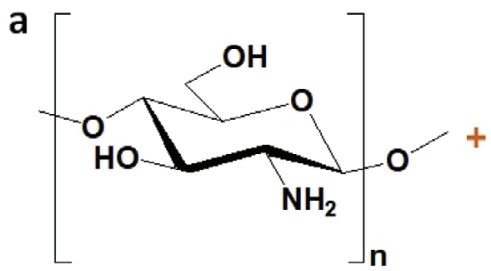

Chitosan chain

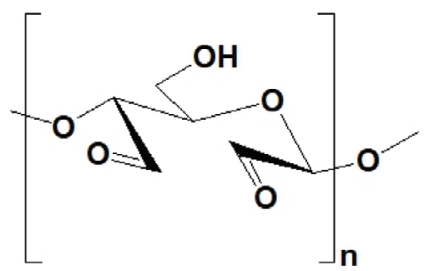

Aldehyde chitosan chain

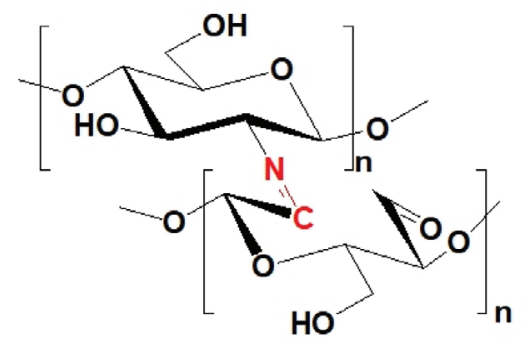

Chitosan/Aldehyde chitosan hydrogel

b

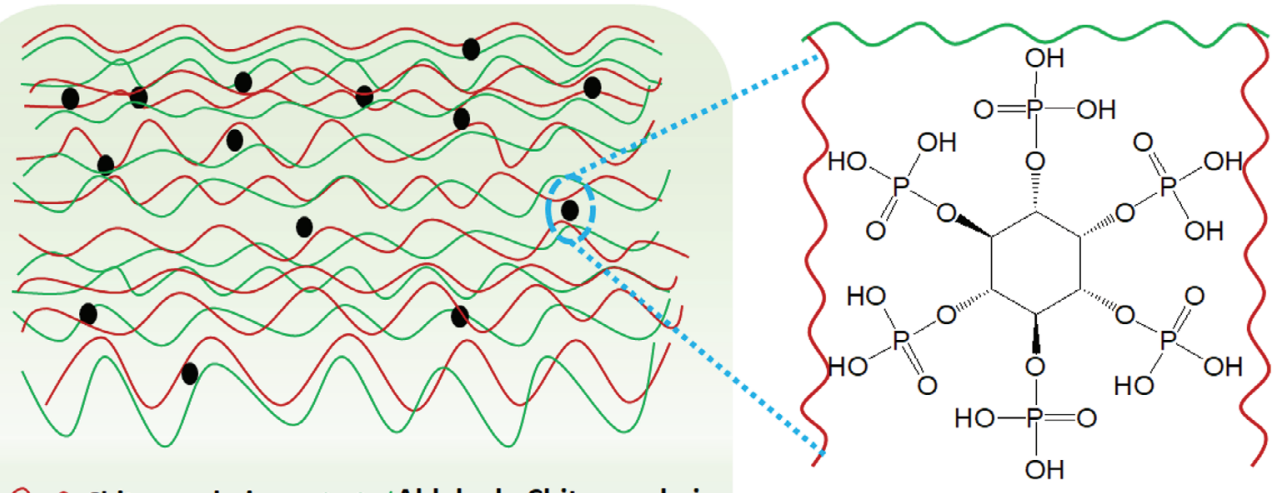

$\sim$ Chitosan chain $\sim$ Aldehyde Chitosan chain

Phytic acid

Figure 3. a) Scheme of formation of $\mathrm{CHI} / \mathrm{ACHI}$ hydrogel from chitosan and aldehyde chitosan and b) schematic representation of the sequential crosslinking in $\mathrm{CHI} / \mathrm{ACHI} / \mathrm{PA}$ hydrogel.

the molecular level, by the fusion of independent, yet interlocking polymer networks. ${ }^{[78-80]}$ Sequential crosslinking comprises of a multistep process. The primary network is formed by the crosslinking of monomers by physical or chemical interactions, followed by a secondary network, which is formed by the polymerization/reaction of the monomers. On the other hand and contrary to this method, simultaneous crosslinking involves a single step process, where direct independent routes are, employed to form an IPN hydrogel, by performing the mixing of monomers and the crosslinking agent. Recently, Chen et al. ${ }^{[81]}$ produced HA-collagen hydrogel by creating simultaneous IPN of independently crosslinked HA and collagen, without chemical bonding between the two networks. However, this process may sometimes lead to the entanglement of monomers or create undesired chemical interactions amongst the networks and this may have an adverse effect on their mechanicalbrk properties. $^{[81,82]}$

\subsection{Sequential Crosslinking}

Recently, Wang et al. ${ }^{[83]}$ successfully employed the sequential crosslinking method to synthesize two layered injectable hydrogels by a two-step reaction. In the first step, chitosan (CHI) was oxidized to aldehyde-chitosan in order to fabricate $\mathrm{CHI} /$ aldehydechitosan first layer (Figure 3a). In order to formulate the second layer, phytic acid was used as a sequential cross-linker, which reacts with the $-\mathrm{NH}_{2}$ groups of $\mathrm{CHI} /$ aldehyde chitosan to form the subsequent layer of CHI/ACHI/PA hydrogel (Figure $3 \mathrm{~b}$ ). The mechanical properties of hydrogel were, enhanced after crosslinking with PA. The mechanical properties of the hydrogels were tested by dynamic time-sweep and frequency-sweep experiments. It was observed that aldehyde played a crucial role in the gelation process. The modulus of CHI/ACHI hydrogels increased with increasing frequency due the crosslink between the aldehyde groups with amino groups of the chitosan. The high- 


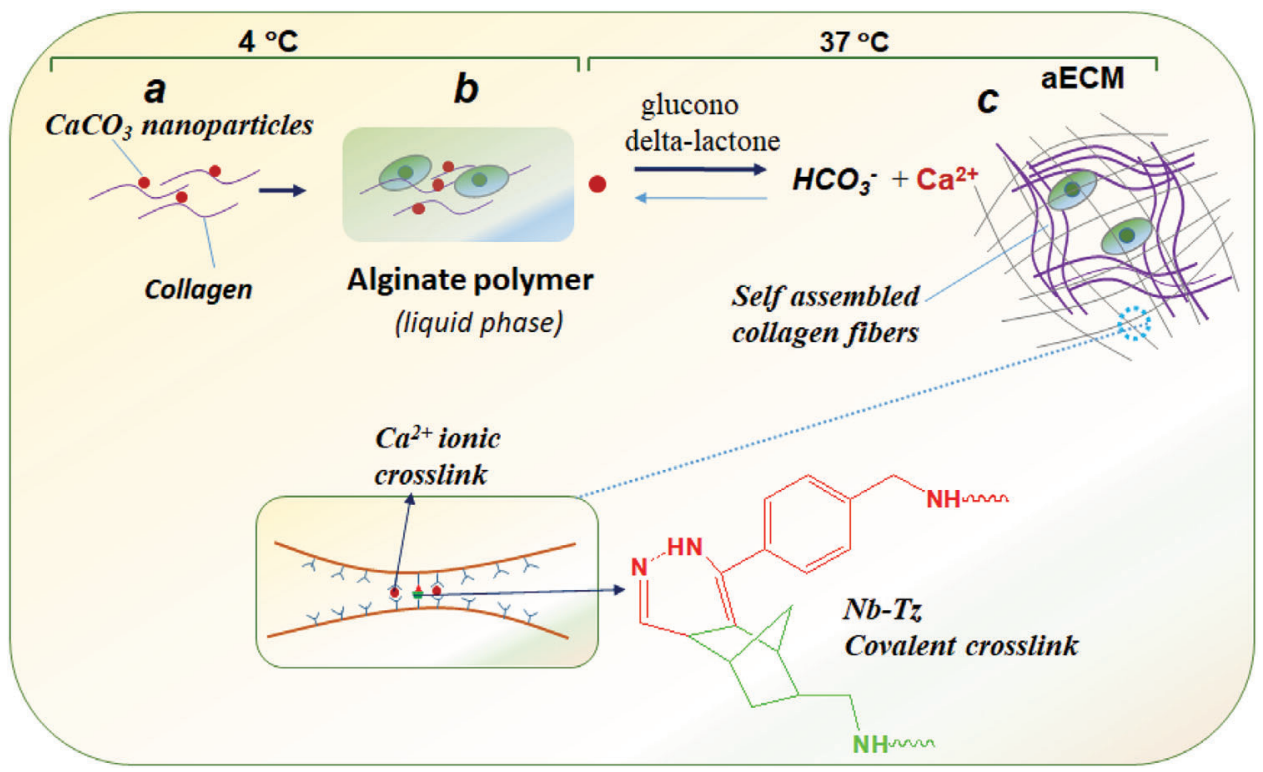

Figure 4. Artificial extracellular matrix (aECM) hydrogel formed by sequential ionic and covalent crosslink: a) $\mathrm{CaCO}_{3}$ nanoparticles (in red) are incorporated into collagen (in purple) to attain a uniform suspension (at $4^{\circ} \mathrm{C}$ ), b) an alginate polymer solution was added to the $\mathrm{collagen}^{-\mathrm{CaCO}_{3}}$ mixture at a neutral $\mathrm{pH},\left(4^{\circ} \mathrm{C}\right)$ and $\left.\mathrm{c}\right)$ in the third step, after the addition of glucono delta-lactone, the mixture was cast in order to form a quick gel at $37^{\circ} \mathrm{C}$. Aligate (containing norbornene $[\mathrm{Nb}]$ and tetrazine $[\mathrm{Tz}]$ ) and self-assembled collagen fibers combine to form an interpenetrating network hydrogel. Alginate (in grey) crosslinks with blocks of glucuronic acid residues to form divalent cations, such as $\mathrm{Ca}^{2+}$ (in red circles), $\mathrm{Nb}$, and Tz provide secondary covalent crosslinks.

est modulus recorded for $\mathrm{CHI} / \mathrm{ACHI}$ was $\approx 80 \mathrm{~Pa}$. In the case of $\mathrm{CHI} / \mathrm{ACHI} / \mathrm{PA}$, the modulus increased with increasing PA concentration and reaction time. The phosphate ions of PA reacted with the amino groups of the CHI/ACHI hydrogel, via a sequential crosslinking process, resulting in an increase of the modulus to $\approx 5000 \mathrm{~Pa}$. Additionally, the two-layered structure, displayed a promising antibacterial activity required for biological applications.

The strategy was redesigned by Mooney and co-workers. ${ }^{[84]}$ This was achieved the by sequential ionic and covalent crosslinking in order to develop the collagen-alginate based artificial extracellular matrix hydrogels. The ionic crosslinking of alginate was applied to alter the crosslinking density and mechanical properties of matrix, while covalent crosslinking was introduced by the incorporation of functionalized alginate containing norbornene $(\mathrm{Nb})$ and tetrazine $(\mathrm{Tz})$. Prior to crosslinking, calcium carbonate nanoparticles were homogeneously distributed into polymer solution in order to initiate ionic crosslinking. Furthermore, the rate of crosslinking was optimized by varying the ratios of $\mathrm{Nb}-\mathrm{Tz}$. The sequence of timescales, between ionic and covalent crosslinks allowed collagen self-assembly to formulate a fibrillar architecture of the native extracellular matrix. The unique architecture of a collagen-alginate hydrogel enabled the formation of tuneable stiffness and viscoelastic properties that are required to regulate cell functions (Figure 4).

Guo et al. ${ }^{[85]}$ employed a similar approach to fabricate s double crosslinking hydrogel by sequential hydrogel bonding, covalent crosslinking, and electrostatic interactions among hyaluronic acid (HA) and poly-L-lysine (PLL). The hydrophilic groups of PLL interacted with HA to form hydrogen bonds. For chemical crosslinking, DMTMM (4-(4,6-dimethoxy-1,3,5-triazin-2-yl)-4- methylmorpholinium chloride) solution was used to activate the carboxyl group of HA chains in order to form an amide linkage amongst the polymer end groups. Subsequently, the single crosslinked (SC) gel was immersed in $\mathrm{NaCl}$ solution in order to create electrostatic interaction (Figure 5a). It was observed that the introduction of electrostatic interactions enhanced the mechanical properties of the hydrogels. The formation of a double network (DN) hydrogel with sequential crosslinking is present in Figure 5a,b.

\subsection{Simultaneous Crosslinking}

Simultaneous crosslinking was successfully, implemented in a multicomponent system consisting of polysaccharides or biocompatible synthetic polymers. Lewandowska-Łańcucka et al., ${ }^{[86]}$ developed multicomponent injectable hydrogels, based on collagen, chitosan, and hyaluronic acid. The amino functionalized silica particles were incorporated into the tri-component matrix of collagen, chitosan, and hyaluronic acid and crosslinked with genipin to form a stable hydrogel (Figure 6). The designing strategy of these gels was proven to be innovative, as it restricted phase separation of mineral particles that is crucial for in-vivo applications (especially in bone tissue engineering). Mata et al. ${ }^{[87,88]}$ investigated the different crosslinking strategies in order to improve the strength and structural integrity of self-assembling systems. They used synthetic (succinimidyl carboxymethyl ester, poly (ethylene glycol) ether tetrasuccinimidyl glutarate and glutaraldehyde) and natural (genipin) crosslinking agents to stabilize biopolymer-peptide of self-assembling systems. It was concluded that genipin exhibited a high de- 
(a)
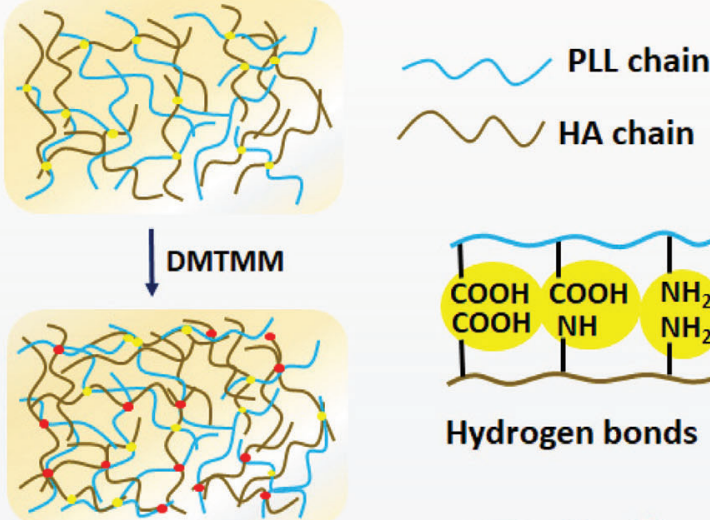

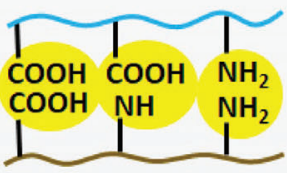

Hydrogen bonds
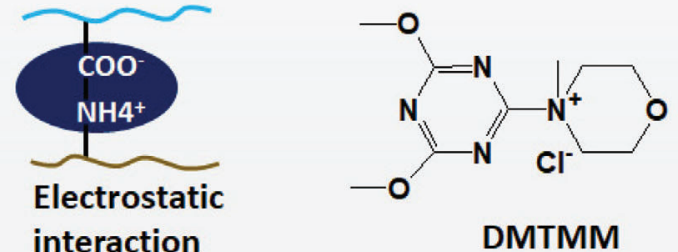

interaction

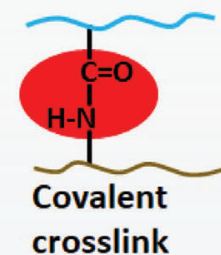

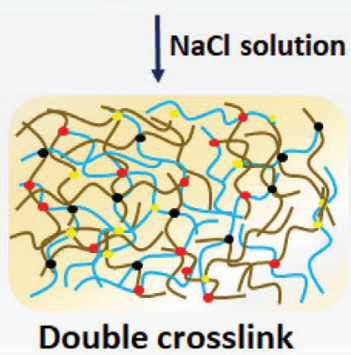

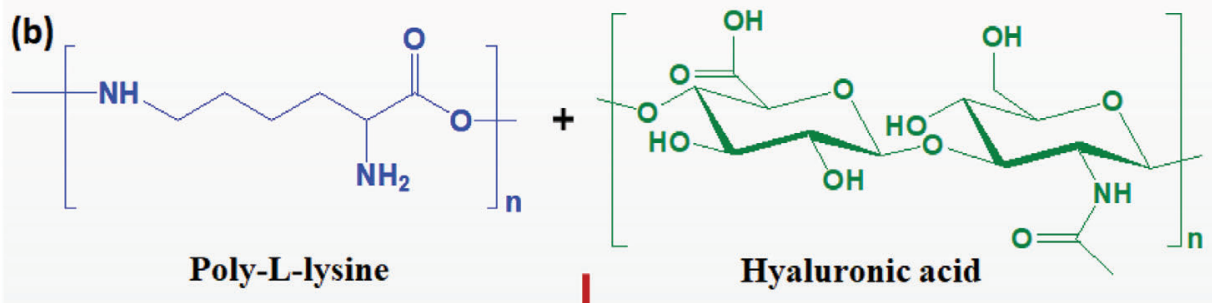

DMTMM

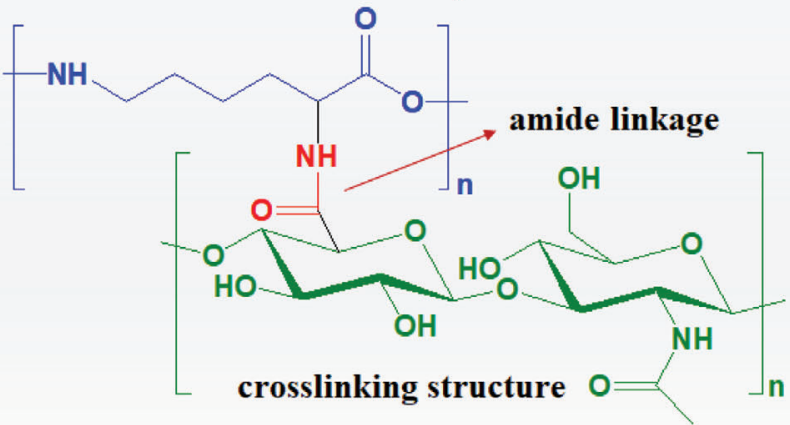

Figure 5. a) Schematic demonstration for the fabrication of double crosslinked hydrogel by hydrogen bonds, covalent crosslink and electrostatic interactions. b) Crosslinking reaction between poly-L-lysine and hyaluronic acid in the presence of DMTMM (4-(4,6-dimethoxy-1,3,5-triazin-2-yl)-4methylmorpholinium chloride). Adapted with permission. ${ }^{[85]}$ Copyright 2021, Elsevier.

gree of resistance in a physiological environment and improved the stability and with a high degree of compatibility when compared to all the other crosslinking agents. The authors suggested genipin as a promising crosslinking agent for improving the stability of self-assembling materials for tissue engineering.
Recently, multicomponent-based antibacterial hydrogels were developed by using aluminum (III) $\left(\mathrm{Al}^{3+} \mathrm{s}\right)$ ions, carboxymethyl chitosan nanoparticles, and polyacrylic acid (PAA) via simultaneous triple physical crosslinking. ${ }^{[8]}$ The aluminum ions and chitosan nanoparticles crosslinked with PAA via hydrogen, coordination, and electrostatic bonds, as shown in Figure 7. The ho- 


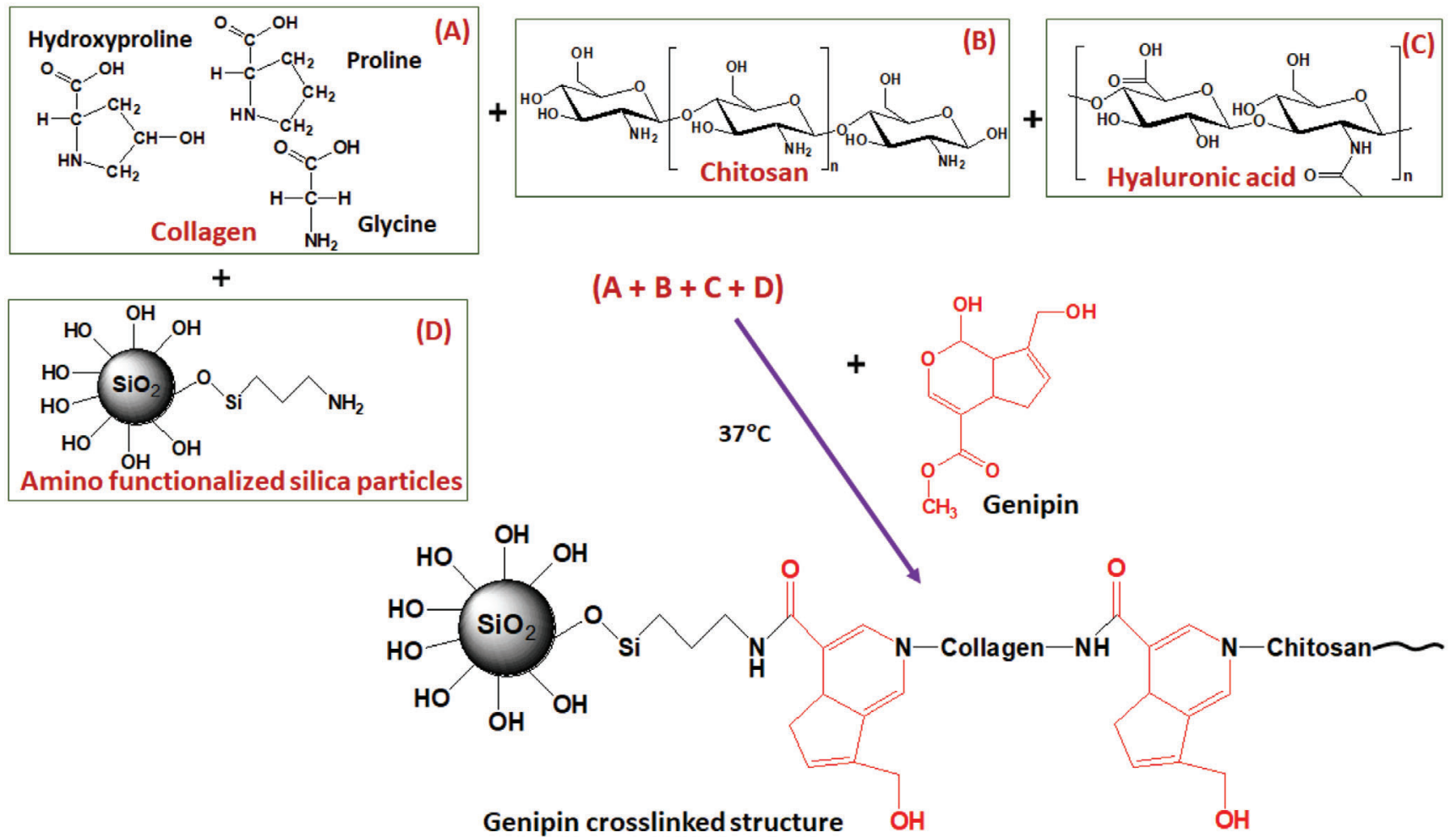

Figure 6. Scheme for the preparation of multicomponent hydrogel via the simultaneous crosslinking. The hydrogels were prepared with aminofunctionalized silica particles, covalently attached to the matrix containing collagen (A), chitosan (B), and hyaluronic acid (C). The genipin solution in the phosphate buffer was used as the crosslinking agent.

mogenous distribution of nanoparticles and the synergy of multiple bonds (coordination, hydrogen, and electrostatic interactions) through the dual crosslinking centers $\left(\mathrm{Al}^{3+}\right.$ and $\mathrm{CMCS}$ nanoparticles), created numerous mediated interactions of network, which efficiently improved the mechanical properties of the hydrogels produced (fracture strength of $\approx 190.9 \mathrm{KPa}$ and elongation of $\approx 1930 \%$ ). Self-healing properties were also observed in these hydrogels, displaying good resistance under harsh environmental conditions. Additionally, the physical crosslinking in the hydrogels contribute some benefits to recycle them via an ecofriendly route.

Despite their many advantages, these polysaccharides also suffer from certain drawbacks, such as low mechanical properties, vague chemical compositions, wide molecular weight distribution, and limited control over physiochemical properties. In order to enhance the mechanical performance, it is essential to design the hydrogels with better homogeneity and integrate a sufficient energy dissipation mechanism in the networks. ${ }^{[0]}$ Multiple strategies have been, implemented, for example, double network, nanocomposite (NC), side ring, etc. Xu et al., ${ }^{[91]}$ reported a distinct approach by constructing a hydrogel by the conjoined network formed by the sharing of linkages of two or three networks (Figure 8). CHI-gelatin and sodium phytate were electrostatically crosslinked in order to formulate a biogenic conjugated network. Sodium phytate with phosphate groups interacted with the amino groups of $\mathrm{CHI} /$ gelatin in order to form two individual networks (Figure 8A,B) and also combined these two networks by connecting them with the amino groups of chitosan and gelatin simultaneously, in order to form a conjoined network. The conjugation of these networks resulted in an equal energy dissipation mechanism and these conjoined networks were linked to effectively, distribute the stress in chitosan-gelatin-phytate system (Figure 8C).

Recently, Panpinit et al. ${ }^{[92]}$ employed the IPN strategy for developing multicomponent hydrogel films from poly(2hydroxyethyl methacrylate-co-acrylamide) (poly(HEMA-co-AM)), PVA and CHI. The primary networks of PVA/CHI were crosslinked with glutaraldehyde (Figure 9a), while the secondary step resulted in the formation of poly(HEMA-co-AM). The secondary networks interpenetrated into the primary networks of PVA/CHI and finally, chemically crosslinked by ethylene glycol dimethacrylate in order to produce the poly(HEMA-coAM)/PVA/CHI IPN hydrogel (Figure 9b). The films developed, showed high mechanical properties with promising antibacterial activity, with a potential application for use in wound dressing.

Recently, Javanbakht et al. ${ }^{[93]}$ reported a new approach for multicomponent reactions (MCR) by crosslinking carboxymethylcellulose (CMC) via a bioactive carboxamide skeleton for drug delivery. The efficiency of the crosslinking on the properties of hydrogel, was studied by modifying the reactant ratios, preparation conditions, and other parameters. The reactants, namely benzaldehyde, ethylenediamine, cyclohexyl isocyanide, and CMC were stirred at $60{ }^{\circ} \mathrm{C}$ for $12 \mathrm{~h}$. The crosslinking mechanism is shown in Figure 10. The crosslinking and functionalization of CMC was performed using Ugi/Passerini MCRs. ${ }^{[94-97]}$ It was observed that the formation of carboxamide-skeletons during the 


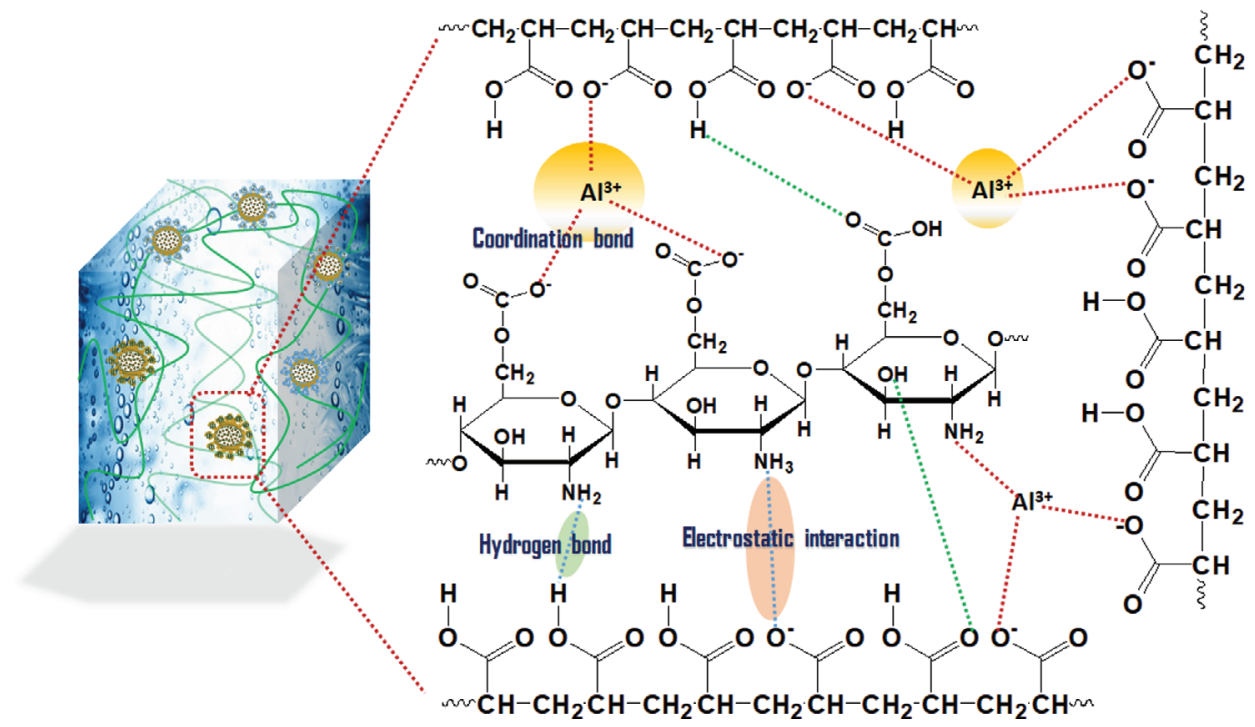

Figure 7. The proposed scheme for the synthesis of triple-physically crosslinked PAA-Al ${ }^{3+}-\mathrm{CMCs}_{\text {h }}$ ydrogel using $\mathrm{Al}^{3+}$ both with $\mathrm{CMCS}$ nanoparticles and PAA main chains. The formation a triple-crosslinking network is formed between $\mathrm{Al}^{3+}, \mathrm{CMCS}$ nanoparticles, and PAA chains through hydrogen bond (in green), coordination bond (in yellow), and electrostatic interactions (in red).
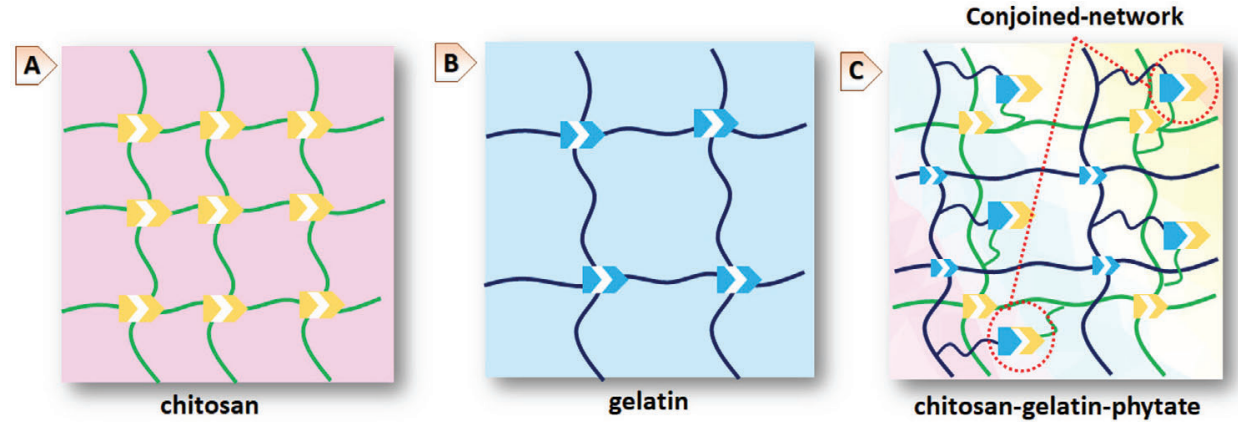

\section{Crosslink of Individual network}

Figure 8. Fabrication of conjoined network hydrogel composed of chitosan, gelatin, and phytate. A) First, a network of chitosan was formed, which effectively consumes energy by the bond rupture and B) the second network of gelatin with similar energy consumption like the first network. C) The two networks of $\mathrm{CHI}$ and gelatin (in sodium phytate solution), entangled with each other to form conjoined-network, which effectively, dissipates stress in the hydrogel. Sodium phytate can interact with amino groups of chitosan/gelatin to form individual networks, also interacts with amino groups of both (chitosan and gelatin) simultaneously, in order to form conjoined network.

crosslinking of CMC, enhanced the bioavailability of CMC multicomponent hydrogels. The hydrogel developed, exhibited a controlled delivery of gentamicin and showed antibacterial activity towards S. aureus and E. coli.

\section{Other Crosslinking Strategies}

Despite the many advantages, the multicomponent systems with different crosslinking mechanisms still have serious disadvantages that severely constrain their usage. Some of the demerits include: a) they release chemical by-products; b) low adhesion; c) some crosslinkers are irritant, allergenic, or toxic; and d) large scale optimization problems, etc. The toxicity of the crosslinking agent is one of the major hurdles in the use of these polymers as injectables, polymer scaffolds, tissue engineering, and drug release systems. The regularly used crosslinking agents, such as glutaraldehyde, polyepoxides, and isocyanates, are highly toxic and susceptible to percolate out into the body after polymer degradation. ${ }^{[98]}$ The solution to reduce these limitations is to design a self-crosslinking or dual crosslinking system. Literature suggests that self-crosslinking can be induced since the method does not require any external crosslinking agent. Examples are, the oxidation of polysaccharides, ${ }^{[99,100]}$ self-crosslinking of oxidized alginate and gelatin for preparing biodegradable polymer scaffold, ${ }^{[101]}$ and self-crosslinked gelatin for controlled release. ${ }^{[102]}$ Others are self-crosslinked hyaluronic acid hydrogels as injectable scaffolds for tissue engineering and cell therapy, ${ }^{[103]}$ injectable hydrogel from alginate dialdehyde and gelatin for the treatment of meniscal repair, ${ }^{[104]}$ the oxidized alginate-gelatin hydrogel for cartilage regeneration, ${ }^{[105]}$ etc. Such strategies can re- 
(a)
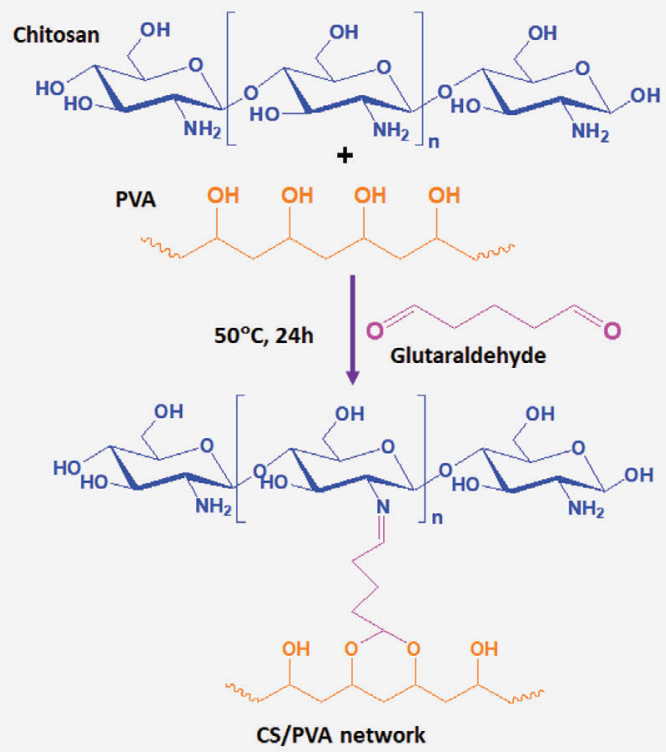

(b)

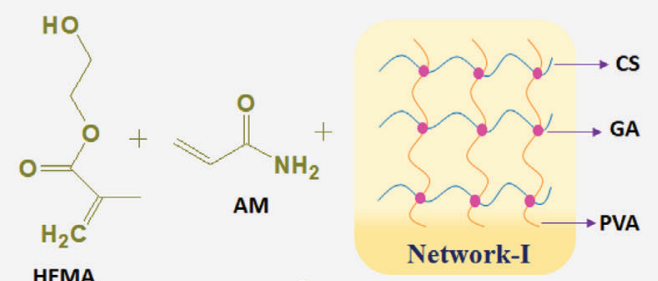

HEMA
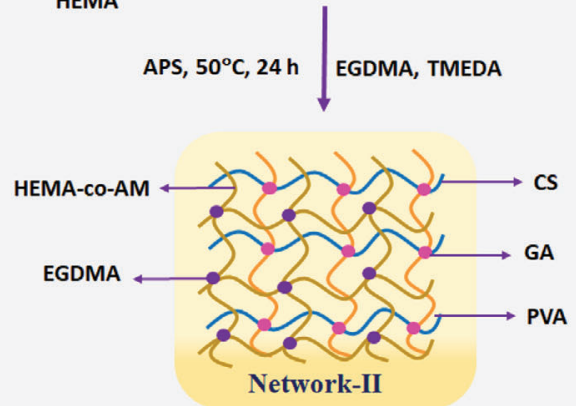

Figure 9. Synthesis of poly(HEMA-co-AM)/PVA/CS hydrogel. a) CS/PVA network: chitosan and PVA were separately dissolved (1\% acetic acid) for $24 \mathrm{~h}$. Subsequently, glutaraldehyde (a crosslinker) was added to the mixed solution and crosslinking reaction was carried out at $50^{\circ} \mathrm{C}$ in order to form primary network of CS/PVA solution. b) Formation of a second poly(HEMA-co-AM)/PVA/CS network: HEMA, AM, EGDMA (crosslinker), TMEDA, and APS (initiators) were added to the primary network of CS/PVA solution and stirred at $50^{\circ} \mathrm{C}$ for $24 \mathrm{~h}$ in order to form the second network of poly(HEMA-co-AM) polymers, interpenetrated in the primary network. Adapted with permission. ${ }^{\text {92] }}$ Copyright 2020, Elsevier.

solve the present difficulties encountered by the application of polysaccharides and their nanoparticles for biomedical applications. However, self-crosslinked hydrogels are inherently limited by low mechanical properties and rapid degradation. In order to overcome these limitations, self-crosslinking is combined with physical crosslinking to form a dual-crosslinking system. This has been an effective method to control the injectability, degradation behavior, self-healing features, and mechanical strength.
Recently, Maiti et al. ${ }^{[100]}$ applied a dual crosslinking strategy by the synergistic combination of physical and self-crosslinking mechanisms in order to synthesize a tricomponent hydrogel based on chitosan, gelatin, and guar gum/oxidized guar gum and crosslinked by $\beta$-glycerophosphate and sodium bicarbonate. The resultant tri-component structure led to the formation of different types of interactive bonds; for instance, chitosan interacted with gelatin via the $\mathrm{C}-\mathrm{O}$ bond formation, $\beta$-glycerophosphate interacted with chitosan units by ionic bond, gelatin interacted with guar gum by $\mathrm{C}-\mathrm{N}$ bond formation and oxidized guar gum by the amide bond formation. Furthermore, it was revealed that the carbonyl group of the oxidized guar gum was engaged in selfcrosslinking with gelatin. The mechanism is schematically presented in Figure 11.

Türk et al. ${ }^{[106]}$ employed the self-crosslinking approach to develop a multicomponent injectable hydrogel by combining nitrogen-doped carbon quantum dots (NCQD), doxorubicin (Dox), and hydroxyapatite (HA) for use in cancer treatment. The hydrogels were fabricated by uniting them via the Schiff base and forming inter-connecting bonds (ionic and H-bonds) among the tricomponents, as shown in Figure 12. The NCQDs/Dox/HA hydrogels developed, demonstrated an in-situ gelation, injectability, and effective drug release. The self-crosslinking confines the use of extra crosslinking agents for binding and thereby, guards the release of any types of harmful by products.

Gao et al. ${ }^{[107]}$ prepared self-crosslinked injectable hydrogels by using Dulbecco's modified eagle's medium (DMEM), induced phenylboronic acid (PBA) modified with hyaluronic acid (by conjugating PBA and diol on HA). The presence of reversible phenylboronic acid esters provided sufficient injectability and self-healing properties. The phenylboronic acid binds to o-diols in tissues, diffuse internally from the tissue, forming a topological structure and the crosslinking of HA-phenylboronic acid, stabilized the topological structure. Due to this crosslinking effect, the gel exhibited a unique shear-dependent and reversible gel-sol transition, ${ }^{[108]}$ with shear thinning phenomenon of the hydrogel that can be fluidized through the gel-sol under shear stress during the injection process and then the gel can be easily inserted at the wound site. The secondary network was created by incorporating PEG or photo-crosslinking to enhance the mechanical stabilities of the hydrogel. The resultant gels formed uniform networks and displayed good adhesion to tissues due to the phenylboronic acid linkage to o-diols, the self-crosslinking of HA-PBA, and the hydrogen bonding in phenylboronic acid (Figure 13).

Issues are often raised when these polysaccharides are interconnected with cells/tissue for biomedical applications. Hydrogels often lack strong adhesion with the adjacent tissues, posing a big challenge for the medical research domains. In order to overcome this challenge, one distinctive attempt was reported by Derkus et al. ${ }^{[109]}$ The group designed multicomponent hydrogels (MCHs) by the self-assembly of hyaluronic acid with peptide amphiphiles (PAs) in order to stimulate osteogenesis and angiogenesis, via an enzymatic crosslinking for bone extracellular matrix. HA was modified with tyramine (HA-Tyr) and amphiphiles were modified to support cell adhesion (RGDS-PA), endothelial vascular organization (Angio-PA), and osteoblastic differentiation (Osteo-PA). In order to enable the multicomponent self-assembly to function adequately, HA-Tyr was reacted with the negatively charged Osteo-PVA and RGDS-PA and subsequently, with the 

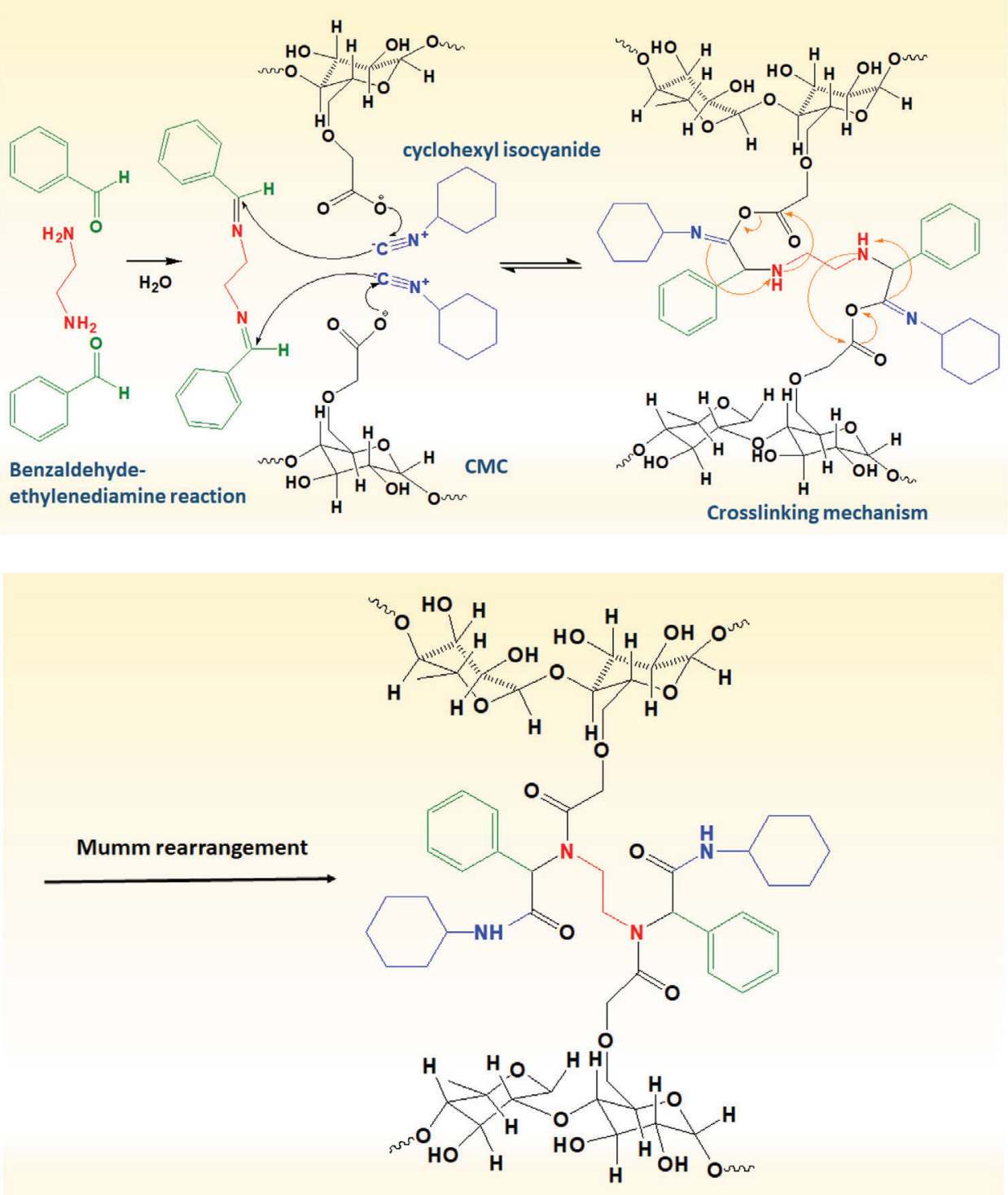

Ugi-Multicomponent reactions

Figure 10. Preparation of CMC-MCC hydrogel and the crosslinking of CMC by the MCR strategy. Adapted with permission. ${ }^{[93]}$ Copyright 2020, Elsevier.

positively charged Angio-PA. The hydrogels developed were successful to stimulate cell adhesion in 2D and 3D setups (Figure 14a,b).

Recently, Nguyen et al. ${ }^{[110]}$ also reported an innovative strategy by interconnecting small interfering RNA (siRNA) to dextran (DEX) hydrogel in order to regulate the effective siRNA delivery for its potential application in tissue regeneration and therapeutics. DEX-MAES containing ester linkages were photopolymerized in order to form a covalently bonded siRNA-DEX-MAES hydrogel. Once the ester groups between siRNA and DEX hydrogel are degraded, the si-RNA, having the $-\mathrm{COOH}$ group, is decoupled from the hydrogels and can be dispersed out (Figure 15). The covalently bonded siRNA to DEX hydrogel is advantageous to control the release of the RNA within the hydrogel with a minimum amount of early burst release. The sRNA released from the hydrogel was mainly governed by the hydrolytic degradation linkages between RNA and DEX hydrogel, independently from the RNA diffusion or bulk degradation. The scheme provided a new approach for regulating nucleic acid delivery via covalent bonds with hydrogels and governs the cellular gene expression, which is a current requirement in biomedical sectors.

There was some degree of curiosity that was generated towards the use of multicomponent, low-molecular weight gels (LMWGs) or supramolecular gels for a wide range of biomedical applications. Adams et al. ${ }^{[111,112]}$ have reported a significant amount of work on the development of multicomponent LMWGs and low molecular weight gelators. ${ }^{[76,113]}$ These gels are typically formed by the self-assembly of small molecules into 1D structures that crosslink to form a network that is capable of immobilizing the solvent. Many efforts have been made to regulate the properties 


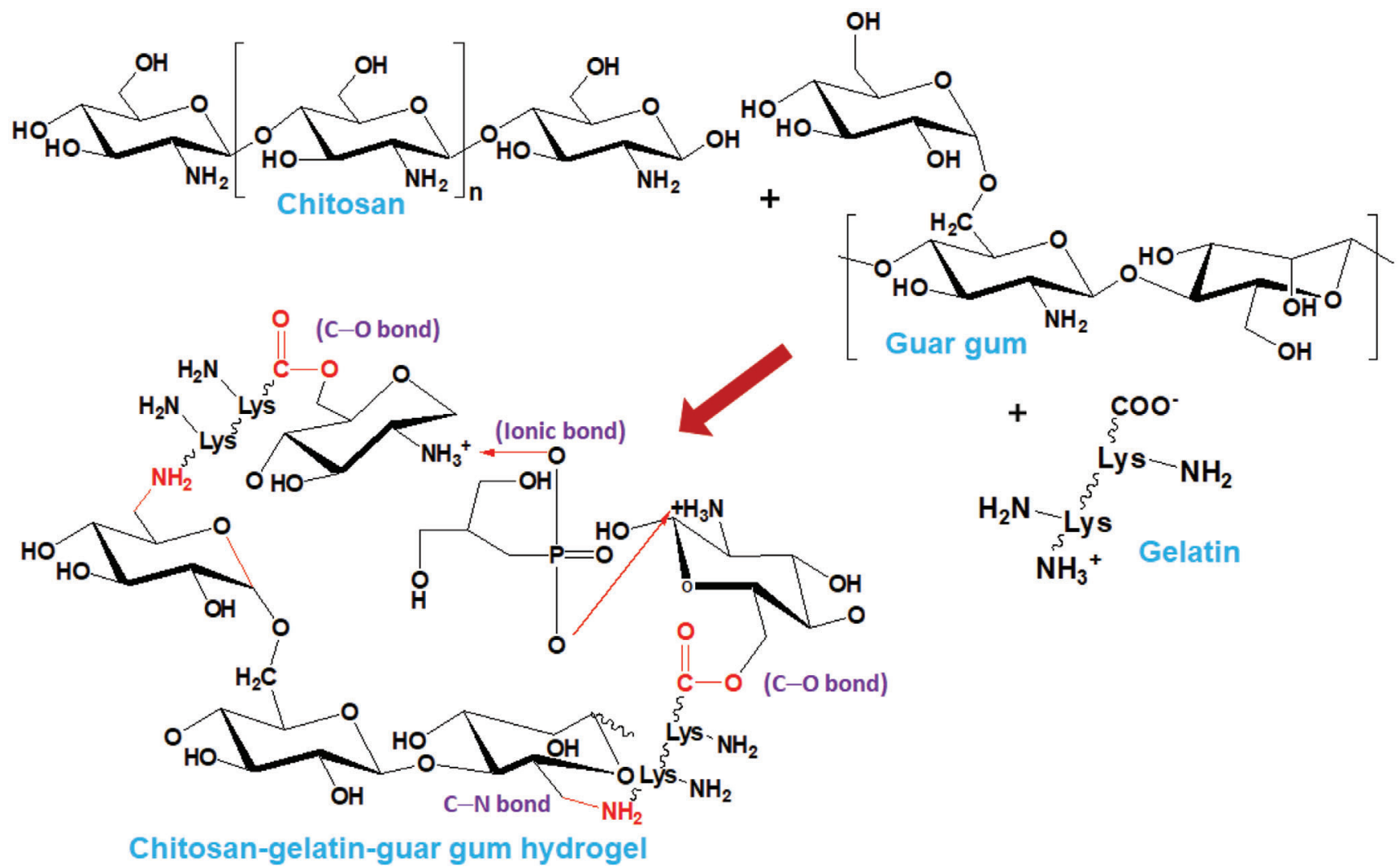

Figure 11. Preparation of chitosan-gelatin-guar gum hydrogel and schematic representation of interactive bonds in the tri-component hydrogel.

of these gels via the molecular engineering of a different set of substrates in order to use them in biomedical researches. ${ }^{[114-116]}$ Of recent, the Adams group proposed a different approach to prepare LMWGs that showed a $\mathrm{pH}$-responsive behavior, involving a strong hydrogen bonding among the components that were permitted to maintain the gel phase for a wide $\mathrm{pH}$ range. The multicomponent hydrogel was composed of two opposite ionizable pendant groups on the different components and it exhibited different phase behaviors and hence, permitted access to two different gel states, both at acidic and basic pHs. They also reported that the programming between the $\mathrm{pH}$-dependent gel states, was possible by the introduction of an autocatalytic urease-urea reaction, with controlled properties. ${ }^{[17]}$

In order to improve the properties of LMWGs, many strategies have been applied to combine it with a polymer gelator (PG) and form hybrid gels in order to maintain the sensitivity of LMWG with the toughness of PG network. Smith et al., ${ }^{[18]}$ demonstrated the first patterned multi-domain gel by combining a LMWG (1,3:2,4-dibenzylidene- $D$-sorbitol-dicarboxylic acid) and a photoinducible polymer gelator (poly(ethylene glycol) dimethacrylate) (PEGDM). One domain contained LMWG, while the patterned region had both the LMWG and polymer gelator networks. The properties varied with regard to the diffusion of small molecules and the gelator networks that can regulate the diffusion rates in order to obtain systems efficiency over controlled release. The materials have found applications in controlled drug release systems and in the development of patterned scaffolds in tissue engineering. ${ }^{[119]}$ In recent work, the same group employed a dif- ferent strategy by replacing PEGDM with alginate (PG) in order to formulate a control over the LMWG assembly. ${ }^{[120]}$ The introduction of alginate, elevated the thermal and rheological properties of the gels and additionally the mechanical properties were tunable based on the alginate concentration.

\section{Crosslinking in Conductive Hydrogels}

Conductive hydrogels $(\mathrm{CH})$ have recently gained increased attention in the medical field due to their applications in bioelectronics, ${ }^{[121]}$ actuators, ${ }^{[55,122]}$ energy storage devices, ${ }^{[123,124]}$ wearable sensors, ${ }^{[125,126]}$ etc. There are mainly two methods for the preparation of these hydrogels, namely: a) by reinforcement of conductive material; and b) by direct polymerization with the designed hydrogel matrix. The conductivity, mechanical, and biological properties of CHs, are strongly, influenced by the types of conducting material (such as: carbon nanotubes, ${ }^{[127,128]}$ ions, graphene oxide, ${ }^{[129,130]}$ and metallic nanoparticles ${ }^{[131,132]}$ ), the particle size, crosslinking method, and the network structures (single/double/triple). The physically crosslinked conductive hydrogels with different structural polymers/inorganic materials, have been widely investigated ${ }^{[60,61]}$ and found to have low tensile properties and tend to deteriorate after a relatively large elongation, resulting in the deformation or rupture of hydrogels. Hence, researchers are focusing more towards developing flexible $\mathrm{CHs}$ in order to overcome the hydrogel deformation issues.

He et al. ${ }^{[133]}$ proposed a multicomponent conductive hydrogel system by combining MXene-cellulose nanocrystals (CNCs), 


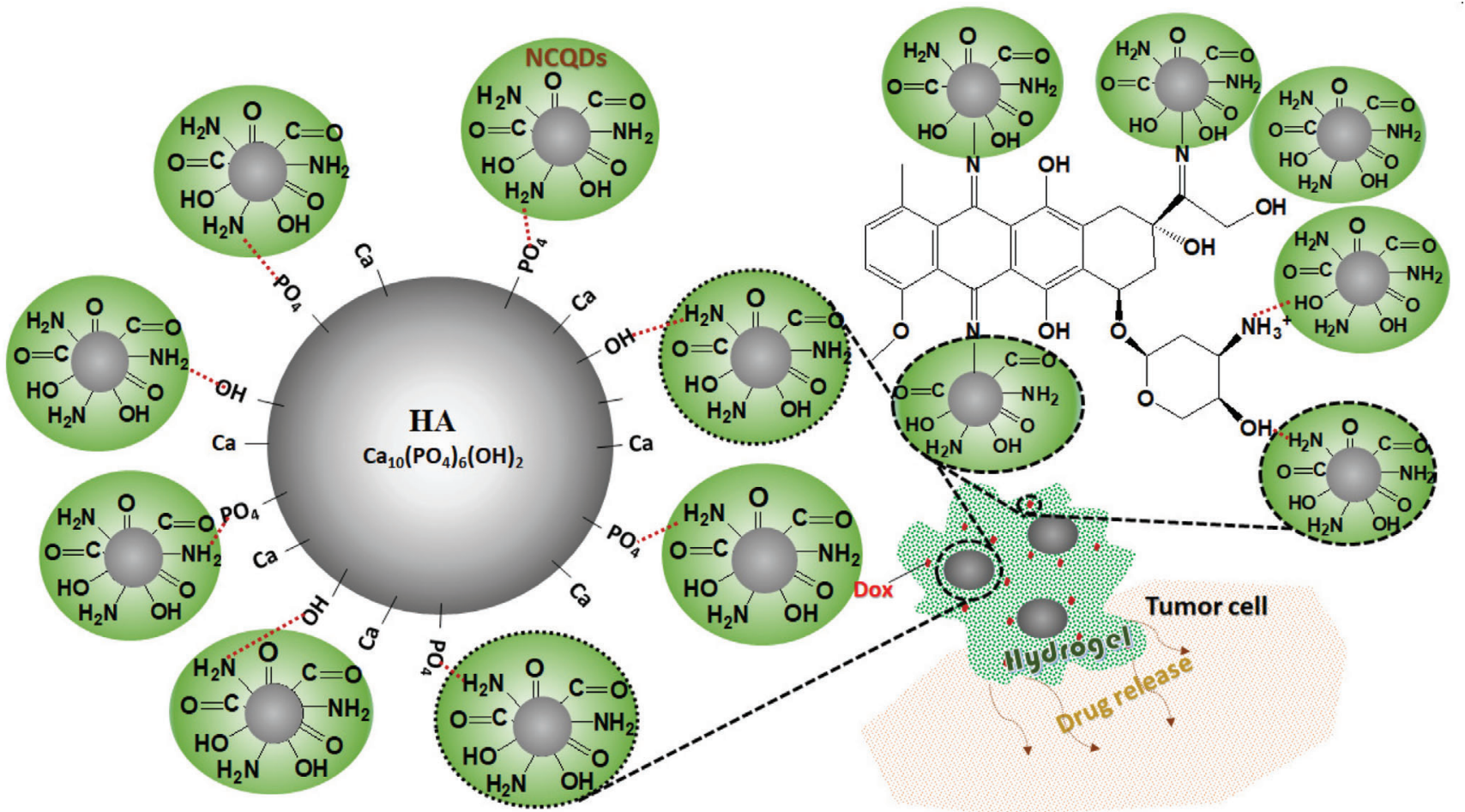

Figure 12. Schematic illustration for the synthesis of NCQDs/Dox/HA hydrogel, formed by self-crosslinking chemical interactions and activated tumor cells release mechanism. HA containing hydroxyl group and having different binding sites, for example, negatively charged phosphate regions and positively charged calcium region on its surface, can penetrate the cell membrane. NCQD (in green) reacts with HA (in grey) and Dox (in red), forming hydrogen bonding and ionic interactions. Adapted with permission. ${ }^{[06]}$ Copyright 2021, Elsevier.
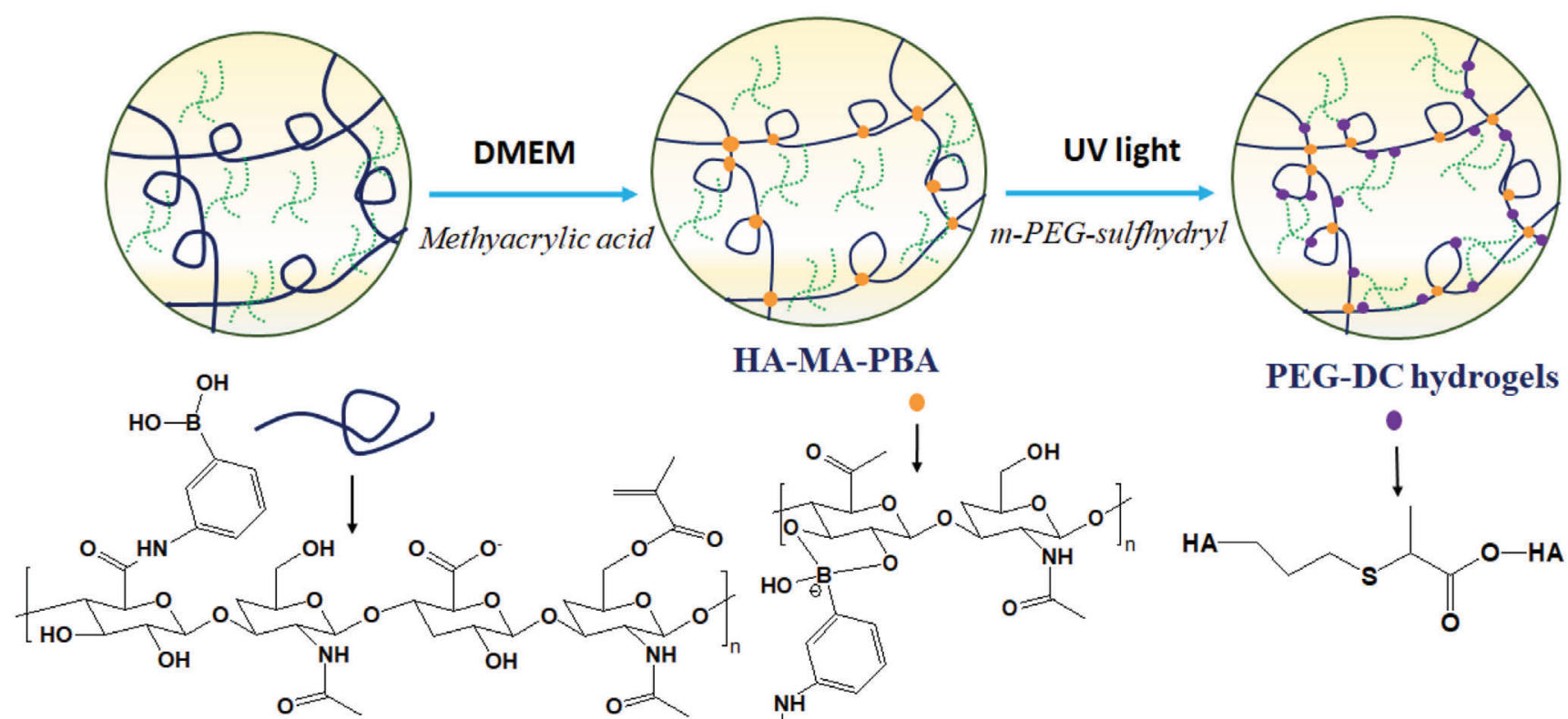

HA-MA-PBA
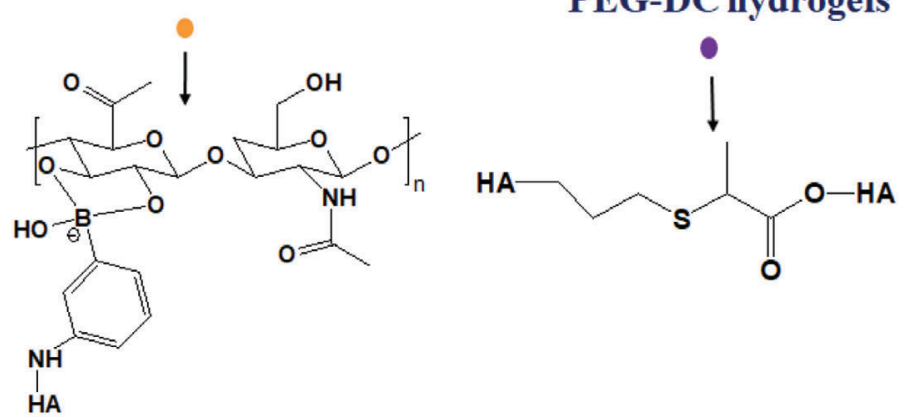

Figure 13. Scheme for the synthesis of PEG double crosslinked hydrogel. The single crosslinked hydrogel of HA-MA-PBA was formed by the reaction between PBA group and the hydroxyl group of HA-MA under the guidance of DMEM. In the second step, PEG double crosslinked hydrogels were obtained

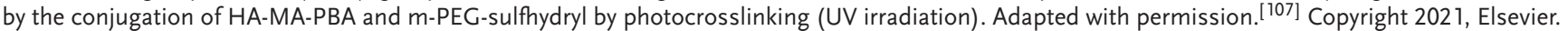




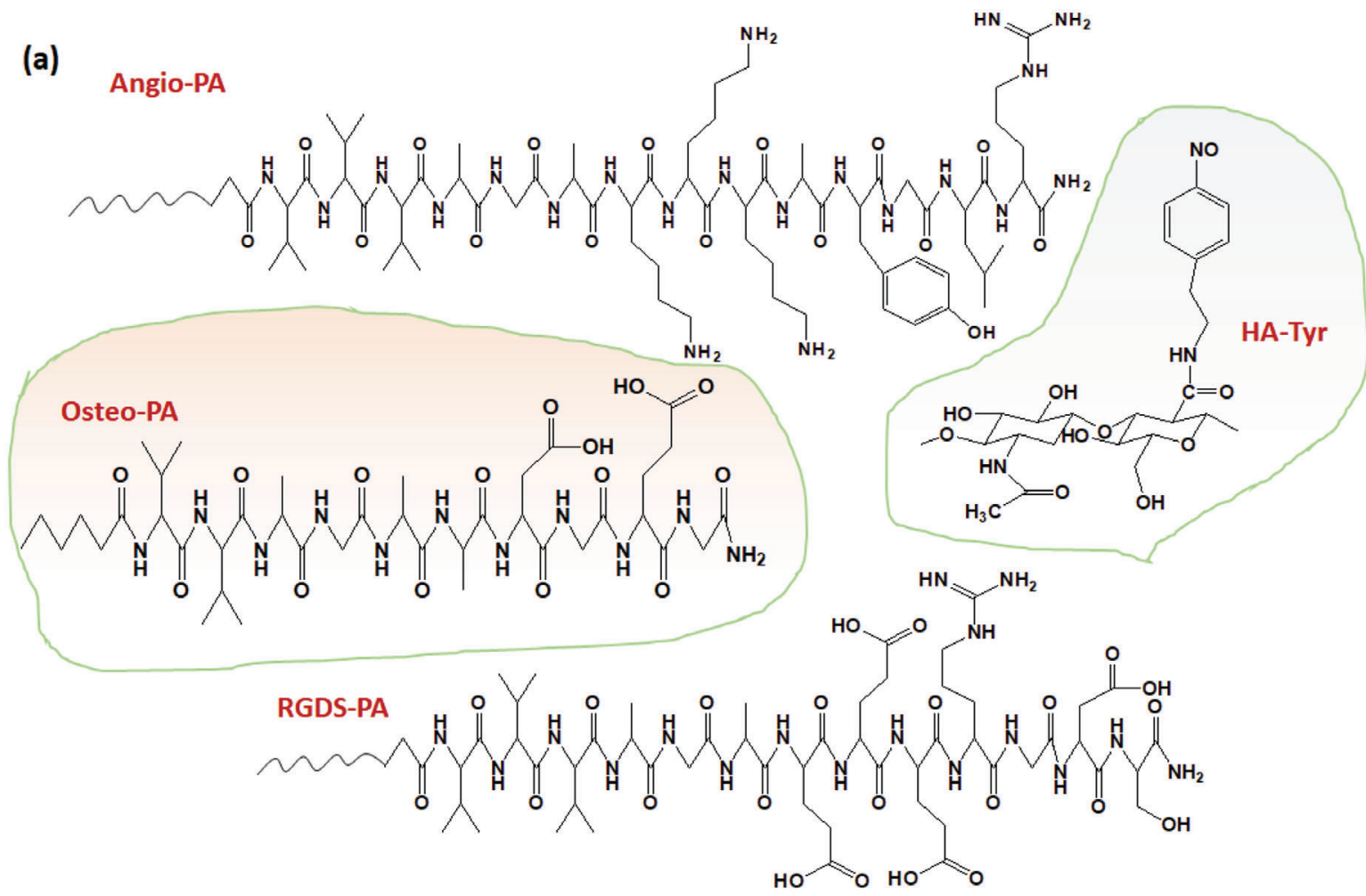

(b)

(2)

RGDS-PA(-)

(3) Anglo-PA(+)
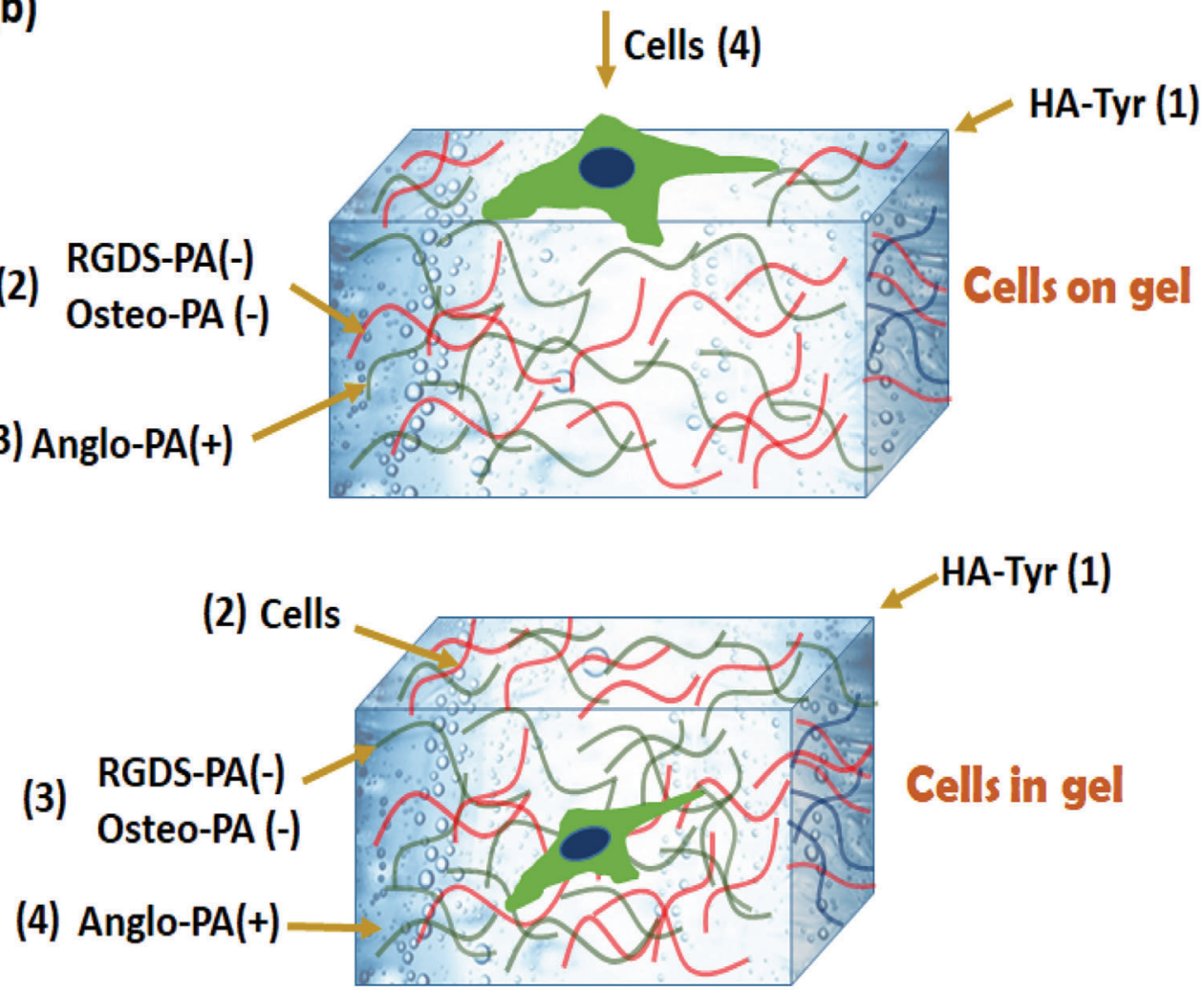

Figure 14. Synthesis of peptide amphiphiles: a) chemical structures of each component used for the gel preparation, angio-PA, RGDS-PA, osteo-PA and HA-tyr. b) Demonstration of 2D and 3D extracellular matrix of multicomponent hydrogels. The numbers in the brackets indicate the order in which they were added for the gel formation. Adapted with permission. ${ }^{[109]}$ Copyright 2020, Elsevier. 


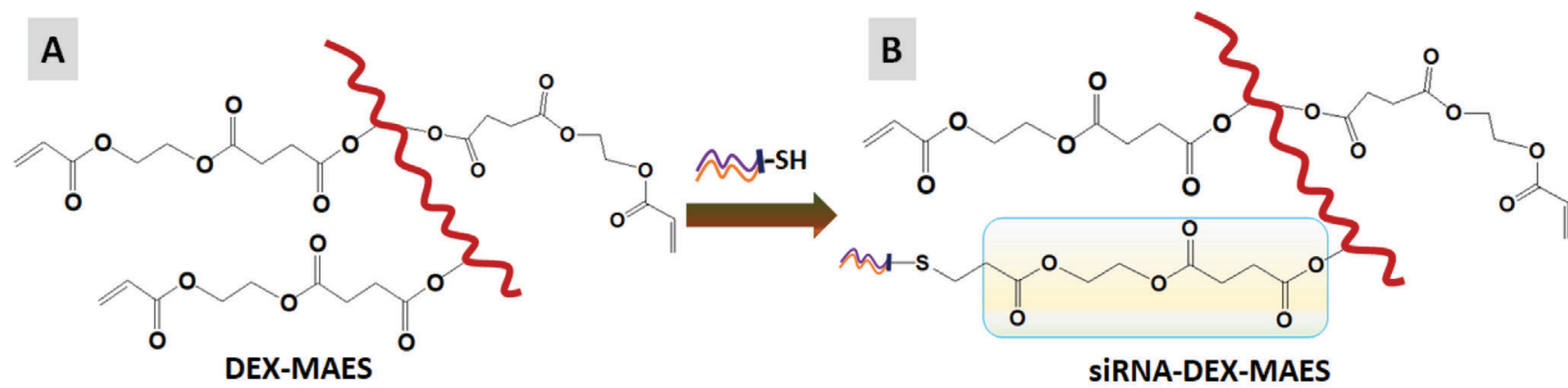

C

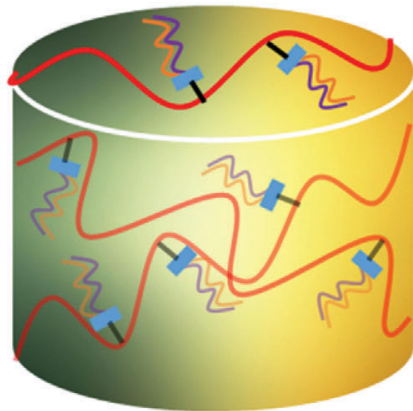

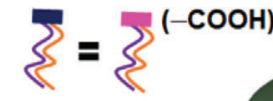

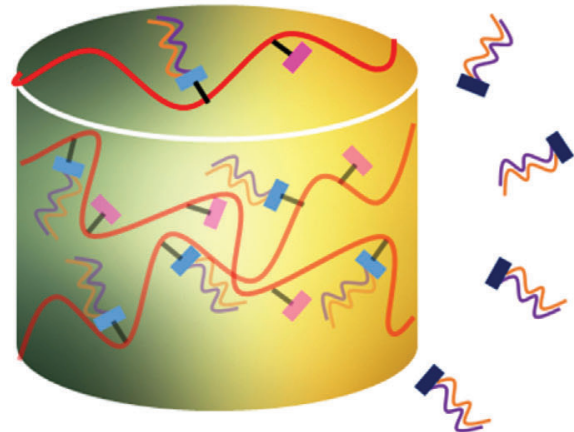

Figure 15. Schematic representation of: A) DEX-MAES, B) siRNA conjugation to DEX-MAES, and C) hydrogel formation (photocrosslinking), siRNA release upon hydrolytic degradation. Adapted from ${ }^{[10]}$ Copyright 2019, The Authors, some rights reserved; exclusive licensee American Association for the Advancement of Science. Distributed under a Creative Commons Attribution NonCommercial License 4.0 (CC BY-NC).

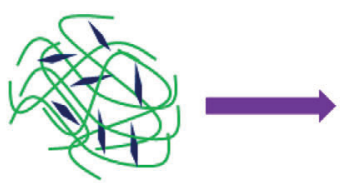

MXene-CNC nanocomposite

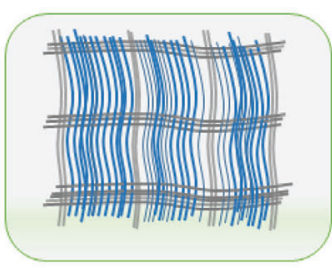

TG-PAM nanocomposite

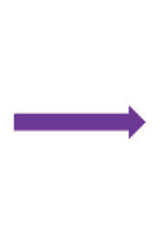

CNCs

I MXene

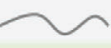

Tamarind gum

MXene-CNC-TG-PAM
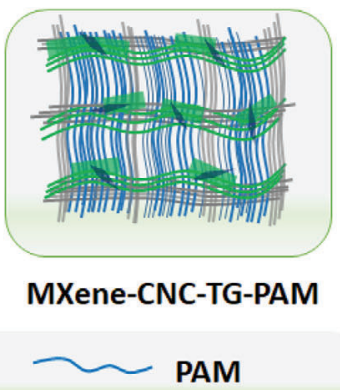

Figure 16. Illustration of the preparation of conductive MXene-CNC-TG-PAM hydrogel. MXene-CNC nanocomposites were incorporated into TG-PAM composites to obtain double network MXene-CNC-TG-PAM hydrogel with ionic TG and covalent PAM network.

tamarind gum (TG), and polyacrylamide (PAM) (Figure 16). The procedure involved the preparation of MXene-CNC suspension (by sonication) in order to form primary network, followed by the addition of acrylamide, aluminum chloride, N, N'-methylenebis acrylamide (crosslinker), and UV light as initiator. The mixed suspension was exposed to UV light in order to form the double network structure. The hydrogels developed were tough and ultra-stretchable ( $\approx 2000 \%$ strain). These improved characteristics were due to formation of double network structure formed by the assimilation of tamarind gum, ionically crosslinked networks, and PAM. In addition, owing to the presence of MXene and mobile ions, the hydrogels exhibited high sensitivity (gauge factor $=5.23$ ) and multiple sensations, ${ }^{[134]}$ such as pressure, strain humidity, and temperature, which are important for practical applications.
Zhang et al. ${ }^{[135]}$ modified the above method by a simple immersion technique in order to develop tri-component ionic hydrogels based on polyacrylic acid, graphene oxide- $\mathrm{Fe}^{+3}$ and chitosan. The first network was formed by the free radical polymerization of acrylic acid, graphene oxide, and chitosan by using $\mathrm{Fe}^{+3}$ as the crosslinking agent. The ionic bonds between $\mathrm{Fe}^{+3}$ and $\mathrm{C}=\mathrm{O}$ groups of polyacrylic acid and graphene oxide formed the primary network, while crosslinked chitosan chains entanglements, resulted in the secondary network. The hydrogels developed, exhibited high sensitivity and ultra-high tensile properties $(\approx 2.7 \mathrm{MPa})$ with excellent conductivity $\left(\approx 13.8 \mathrm{Sm}^{-1}\right)$. The technique was further modified by Wang et al. ${ }^{[136]}$ in order to extend its use in biomedical ionic sensors. They introduced the hydrogel-network constrained-polymerization technique in order to fabricate a semi-interpenetrating ionic conductive hydro- 


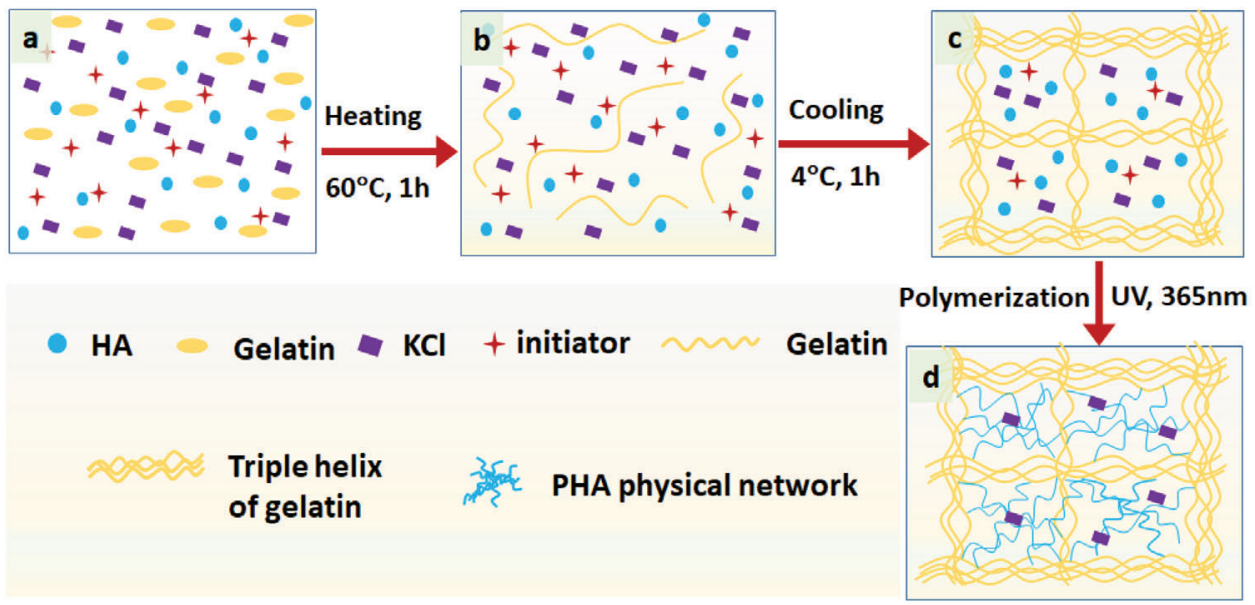

Figure 17. Synthesis of $\mathrm{PHA}$ /gelatin/glycerol conductive hydrogel. a) Gelatine, $\mathrm{HA}, \mathrm{KCl}$, and $\mathrm{HA}$ were added with water and glycerol in a reactor, stirred at $60^{\circ} \mathrm{C}$ for $1 \mathrm{~h}$ (under $\mathrm{N}_{2}$ atmosphere), b) solution was injected into a glass mold and stored at $4{ }^{\circ} \mathrm{C}$ for $1 \mathrm{~h}$, and c) photoinitiated polymerization was conducted under UV light (wavelength $365 \mathrm{~nm}$ ) for $1 \mathrm{~h}$, to obtain PHA/gelatin/glycerol conductive hydrogel.

gel composed of a covalently crosslinked network, intermolecular hydrogen bonding, and electrostatic interactions between PAArandom poly(1-butyl-3-vinylimidazole tetrafluoroborate) and the scattered polyethylene oxide network. The hydrogels showed high stretchability $(\approx 300 \%)$, compression $(\approx 85 \%)$, and displayed fast recovery upon release of external force thereby enabling their use in biomedical ionic sensors.

So far, the conductive hydrogels discussed above are significantly utilized in the medical field due to their biocompatibility, intrinsic flexibility, good conductivity, and self-recovery. However, these gels tend to freeze inevitably at sub-zero temperatures, leading to a gradual decline in their mechanical and electrical properties. In order to overcome these drawbacks, Yang et al. ${ }^{[63]}$ developed the freezing tolerant supramolecular conductive hydrogels composed of poly(N-hydroxymethyl acrylamide) (PHA), gelatin, and glycerol, via a one-pot synthesis. The unique combination was formulated around the interactions between gelatin and PHA network in order to create a double network PHA/gelatin/glycerol conductive hydrogel. Gelatin forms the physical crosslinked network due to a triple-helix structure by heating and cooling processes, while PHA chains form the self-crosslink (photopolymerization), as well as its interaction with gelatin (Figure 17). The hydrogels exhibited anti-freezing resistance, high tensile strength, fast self-recovery, and outstanding fatigue resistance and high ionic conductivity.

Among the group of conjugated polymers, poly(3,4ethylenedioxythiophene):poly(styrenesulfonate) (PEDOT:PSS) has emerged as the most promising material for electrochemical device applications. It provides a promising interface with biological tissues for sensing and stimulation, owing to their favorable electrical and mechanical properties. Doped PEDOT:PSS has been explored for electrodes thermoelectrics and biosensing. While existing methods mostly blend PEDOT:PSS with other compositions, for example, non-conductive polymers, the blending can compromise the resultant hydrogels' mechanical or electrical properties. Lately, PEDOT:PSS has also emerged as an efficient NIR photothermal therapy agent due to its high photothermal conversion efficiency, exceptional photostability, and good biocompatibility. ${ }^{[137]}$

Recently, Lu et al. ${ }^{[138]}$ synthesized pure PEDOT:PSS hydrogels via the interconnected networks of PEDOT:PSS nanofibrils by simple mixing of volatile dimethyl sulfoxide into an aqueous solution of PEDOT:PSS, followed by dry-annealing and rehydration. The gels exhibited high electrical conductivity, stretchability, superior mechanical properties, and tunable swelling properties in wet physiological environments. In another interesting study, Lim and co-workers ${ }^{[139]}$ developed a flexible PEDOT:PSSbased sensor that can measure the amount of salt ions in sweat released from human body. Abedi et al. ${ }^{[140]}$ reported the fabrication of chitosan scaffolds that contained PEDOT:PSS by electrospinning, for application in cardiac tissue engineering. The authors observed that by increasing the PEDOT:PSS content, up to $1 \mathrm{wt} \%$, resulted in, 30-40\% reduction of fiber diameter and increase in electrical conductivity by 100 fold.

More recently, our group ${ }^{[141]}$ reported a simple and costeffective method of synthesizing conductive triple network hydrogels by using agarose, PVA, PAM, and PEDOT:PSS. The onepot synthesis of hydrogel (Ag-PAM-PVA) involved the physicalchemical crosslinking of a unique combination of hydrogenbonded agarose, combined with chemically-crosslinked PAM and PVA network. Agarose formed the primary network at room temperature by the physical crosslinking route, while the solution was chemically crosslinked by UV-polymerization in order to form the second PAM network and later cured in order to form the third PVA network, as shown in Figure 18. Later on, a simple mixing of PEDOT:PSS suspension with the precursor solution of the polymer matrices, permitted the ease of incorporation of PEDOT:PSS into the Ag-PAM-PVA polymer matrix and thus, a simple fabrication of conductive hydrogels was achieved. The proposed crosslinked triple network provided a solution for mechanical strength issues that are related to the practical usage of these hydrogels for advanced biomedical applications.

The tensile and compression properties of hydrogels were enhanced at low PEDOT:PSS concentration, that is, at 0.0325 and $0.065 \mathrm{wt} \%$ and these properties, declined at higher concentra- 

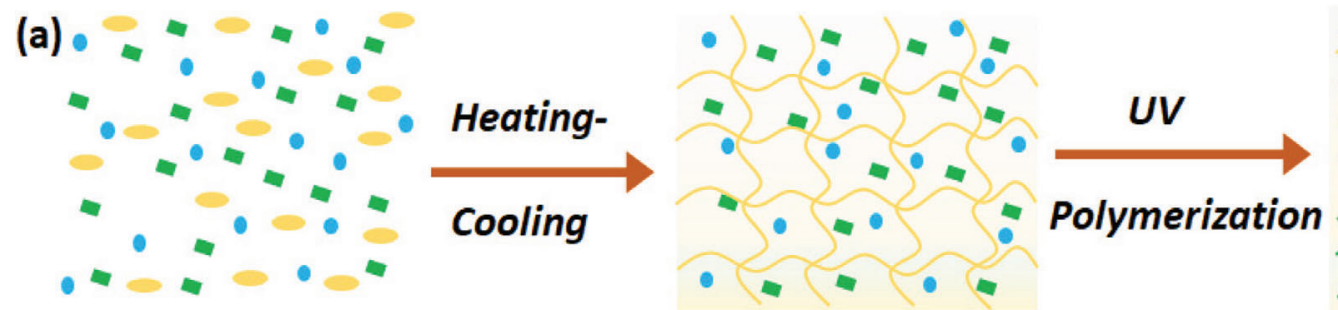

$\mathrm{Ag}-\mathrm{SN}$

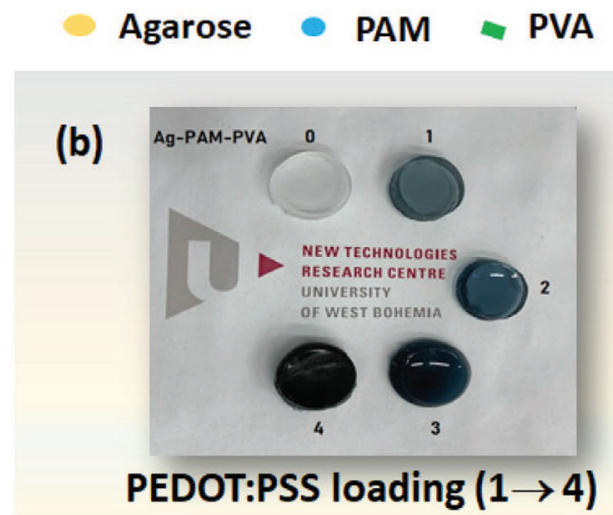

(c)

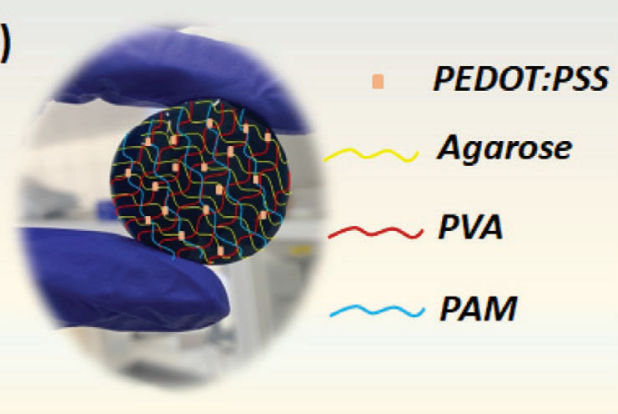

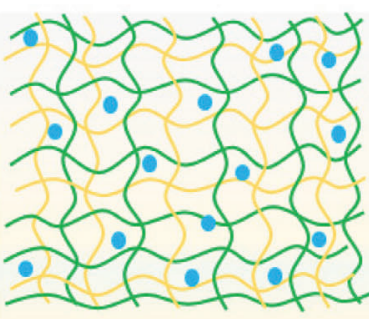

Ag-PAM DN

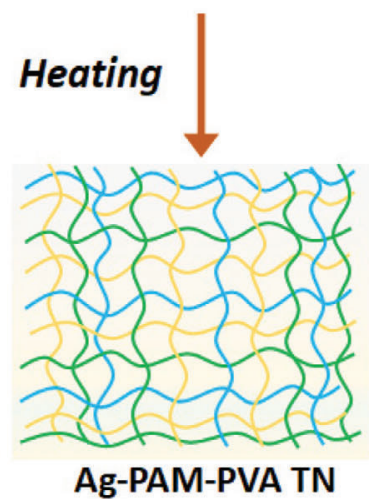

Figure 18. a) Preparation of triple network hydrogels (agarose single network, Ag-PAM double network, and Ag-PAM-PVA triple network), b) photograph of hydrogels with different concentration of PEDOT:PSS, and c) conductive hydrogel of triple network structure with schematic illustration.

tions. The PEDOT:PSS incorporation had a strong effect on the swelling capacity, which deteriorated progressively from $216 \%$ to $155 \%$, when the concentration was increased from $0 \%$ to $0.26 \%$. The structural unity, which was created between the three networks, tended to collapse with the addition of higher concentration of PEDOT:PSS. The results established the fact that uniting three networks resulted in tough and flexible conductive hydrogels with better properties compared to single and double network hydrogels.

\section{Conclusion and Perspective}

This review has highlighted the current ( $<3$ years) crosslinking strategies that have been adopted in the development of multicomponent hydrogel systems for biomedical applications. The influence of crosslinking on polysaccharides, the combination of natural and synthetic polymers and multiple components have been succinctly, discussed. Various approaches that are related to crosslinking (sequential and simultaneous), networks (single, double, and triple network), have been highlighted and the chemistries of multicomponent systems, have been reviewed in details by considering their usage in various medical and tissue engineering aspects. Multicomponent-based conducting hydrogels (dopant PEDOT:PSS) that are widely used in biosensors, actuators, medical devices, and tissue engineering, have also been highlighted. The efficiency of the conductivity of the hydrogels in question and their applications, depend on the structural bonding and stability between the dopant and the designed polymer matrix. The optimizations of the conductivities and the tensile properties of hydrogels are critical for the envisaged practical applications. These applications are mainly focused on soft robotics and soft human tissues that mimic muscles, tendons, and skin, which require high mechanical strength. Different crosslinking strategies have been adopted to formulate multicomponent hydrogels, yet it is still difficult to concretely, specify a particular strategy that can be adopted, universally. Primarily, it depends on the characteristic features of the individual components, their chemical structure, properties, bonding ability, and their biocompatibility, which have direct effects and the possible attendant consequences on the resultant hydrogels. Therefore, the unification of such a complex system requires more research and hence, by considering the domain of biomedical research, natural polymers are the promising candidates. Hence, the designing of appropriate multicomponent hydrogels/films with complete polysaccharides or natural polymers, is a new challenge in biomedical research that must be critically, engaged. The presence of different functionalities in multicomponent systems, provides innovate opportunities that can enable the design of new hydrogels/films for emerging biomedical applications.

\section{Acknowledgements}

The work was supported from the European Regional Development FundProject, Application of Modern Technologies in Medicine and Industry" (No. CZ.02.1.01/0.0/0.0/17_048/0007280).

\section{Conflict of Interest}

The authors declare no conflict of interest.

\section{Keywords}

crosslinking, hydrogels, hydrogen bonding, multicomponent, network formation, self-assembly 
Received: June 7, 2021

Revised: September 9, 2021

Published online:

[1] O. Wichterle, D. Lim, Nature 1960, 185, 117

[2] F. Lim, A. M. Sun, Science 1980, 210, 908.

[3] H. Gin, B. Dupuy, A. Baquey, C. H. Baquey, D. Ducassou, J. Microencapsulation 1990, 7, 341.

[4] A. Bin Imran, K. Esaki, H. Gotoh, T. Seki, K. Ito, Y. Sakai, Y. Takeoka, Nat. Commun. 2014, 5, 5124.

[5] J. P. Gong, Soft Matter 2010, 6, 2583.

[6] D. Y. Ko, U. P. Shinde, B. Yeon, B. Jeong, Prog. Polym. Sci. 2013, 38, 672.

[7] J. Kopecek, Biomaterials 2007, 28, 5185.

[8] S. Merino, C. Martin, K. Kostarelos, M. Prato, E. Vazquez, ACS Nano 2015, 9, 4686.

[9] S. Mohammadi, S. Mohammadi, A. Salimi, Talanta 2021, 224, 121895.

[10] I. J. Sabino, R. Lima-Sousa, C. G. Alves, B. L. Melo, A. F. Moreira, I. J. Correia, D. de Melo-Diogo, Int.J. Pharm. 2021, 600, 120510.

[11] S. Tavakoli, M. Kharaziha, S. Nemati, A. Kalateh, Carbohyd. Polym 2021, 251, 117013

[12] F. Topuz, A. Nadernezhad, O. S. Caliskan, Y. Z. Menceloglu, B. Koc, Carbohydr. Polym. 2018, 201, 105.

[13] E. Brynda, M. Stol, V. Chytry, I. Cifkova, J. Biomed. Mater. Res. 1985 19, 1169

[14] E. M. Ahmed, J. Adv. Res. 2015, 6, 105.

[15] A. M. Mathur, S. K. Moorjani, A. B. Scranton, J. Macromol. Sci., Rev. Macromol. Chem. 1996, 36, 405.

[16] K. R. Park, Y. C. Nho, Radiat. Phys. Chem. 2003, 67, 361.

[17] J. M. Rosiak, P. Ulanski, L. A. Pajewski, F. Yoshii, K. Makuuchi, Radiat. Phys. Chem. 1995, 46, 161.

[18] E. M. Moore, J. L. West, Regener. Eng. Transl. Med. 2019, 5, 167.

[19] S. Burkert, T. Schmidt, U. Gohs, H. Dorschner, K. F. Arndt, Radiat. Phys. Chem. 2007, 76, 1324.

[20] R. Ravichandran, C. Astrand, H. K. Patra, A. P. F. Turner, V. Chotteau, J. Phopase, RSC Adv. 2017, 7, 21068.

[21] I. V. Yannas, E. Lee, D. P. Orgill, E. M. Skrabut, G. F. Murphy, Proc. Natl. Acad. Sci. USA 1989, 86, 933.

[22] J. Glowacki, S. Mizuno, Biopolymers 2008, 89, 338.

[23] C. K. Kuo, P. X. Ma, Biomaterials 2001, 22, 511.

[24] J. Lee, M. J. Cuddihy, N. A. Kotov, Tissue Eng., Part B 2008, 14, 61.

[25] A. Mol, M. I. van Lieshout, C. G. D. Veen, S. Neuenschwander, S. P. Hoerstrup, F. P. T. Baaijens, C. V. C. Bouten, Biomaterials 2005, 26, 3113.

[26] I. M. Garnica-Palafox, F. M. Sanchez-Arevalo, Carbohyd. Polym. 2016, 151, 1073.

[27] D. A. Gyles, L. D. Castro, J. O. C. Silva, R. M. Ribeiro-Costa, Eur. Polym. J. 2017, 88, 373.

[28] K. Chae, W. Y. Jang, K. Park, J. Lee, H. Kim, K. Lee, C. K. Lee, Y. Lee, S. H. Lee, J. Seo, Sci. Adv. 2020, 6, eabb0025.

[29] D. Sharma, D. Saha, B. K. Satapathy, J. Mech. Behav. Biomed. Mater. 2021, 116, 104331.

[30] R. K. Rajan, S. Chandran, H. V. Sreelatha, A. John, R. Parameswaran, ACS Appl. Bio Mater. 2020, 3, 1924.

[31] A. J. T. Teo, A. Mishra, I. Park, Y. J. Kim, W. T. Park, Y. J. Yoon, ACS Biomater. Sci. Eng. 2016, 2, 454.

[32] M. E. Freeman, M. J. Furey, B. J. Love, J. M. Hampton, Wear 2000, $247,129$.

[33] X. Gu, Y. F. Xu, S. Li, Z. Z. Wang, Q. H. Meng, J. J. Yu, ACS Appl. Polym. Mater. 2021, 3, 3519 .
[34] S. J. Jhaveri, M. R. Hynd, N. Dowell-Mesfin, J. N. Turner, W. Shain, C. K. Ober, Biomacromolecules 2009, 10, 174.

[35] Y. Ohm, C. F. Pan, M. J. Ford, X. N. Huang, J. H. Liao, C. Majidi, Nat. Electron. 2021, 4, 185.

[36] J. Yeom, A. Choe, S. Lim, Y. Lee, S. Na, H. Ko, Sci. Adv. 2020, 6, eaba5785.

[37] K. Liu, X. F. Pan, L. H. Chen, L. L. Huang, Y. H. Ni, J. Liu, S. L. Cao, H. P. Wang, ACS Sustainable Chem. Eng. 2018, 6, 6395.

[38] R. N. Xu, S. H. Ma, P. Lin, B. Yu, F. Zhou, W. M. Liu, ACS Appl. Mater. Interfaces 2018, 10, 7593

[39] Y. Y. Dou, Z. P. Wang, W. Q. He, T. J. Jia, Z. J. Liu, P. C. Sun, K. Wen, E. L. Gao, X. Zhou, X. Y. Hu, J. J. Li, S. L. Fang, D. Qian, Z. F. Liu, Nat. Commun. 2019, 10, 5293.

[40] S. Kalasin, P. Sangnuang, P. Khownarumit, I. M. Tang, W. Surareungchai, ACS Biomater. Sci. Eng. 2020, 6, 1247.

[41] M. Fredell, M. B. Hamill, Eye 2019, 33, 858

[42] Y. Ko, J. Kim, D. Kim, Y. Yamauchi, J. H. Kim, J. You, Sci. Rep. 2017, 7, 2282.

[43] O. Catanzano, G. G. d'Ayala, A. D' Agostino, F. Di Lorenzo, C. Schiraldi, M. Malinconico, R. Lanzetta, F. Bonina, P. Laurienzo, Carbohyd. Polym. 2021, 264, 117987.

[44] T. Lin, Q. W. Bai, J. Peng, L. Xu, J. Q. Li, M. Zhai, Carbohyd. Polym. 2018, 200, 72.

[45] J. J. J. Long, A. E. Etxeberria, C. Kornelsen, A. V. Nand, S. Ray, C. R. Bunt, A. Seyfoddin, ACS Appl. Bio Mater. 2019, 2, 2766.

[46] N. Masood, R. Ahmed, M. Tariq, Z. Ahmed, M. S. Masoud, I. Ali, R. Asghar, A. Andleeb, A. Hasan, Int. J. Pharm. 2019, 559, 23.

[47] X. Qi, W. Pan, X. Tong, T. Gao, Y. Xiang, S. You, R. Mao, J. Chi, R. Hu, W. Zhang, H. Deng, J. Shen, Carbohydr. Polym. 2021, 264, 118046.

[48] H. Xue, L. C. Hu, Y. Xiong, X. W. Zhu, C. Y. Wei, F. Q. Cao, W. Zhou, Y. Sun, Y. Endo, M. F. Liu, Y. Liu, J. Liu, A. Abududilibaier, L. Chen, C. C. Yan, B. B. Mi, G. H. Liu, Carbohyd. Polym. 2019, 226, 115302.

[49] Y. M. Kook, S. Hwang, H. Kim, K. J. Rhee, K. Lee, W. G. Koh, Sci. Rep. 2020, 10, 20321.

[50] D. R. Griffin, M. M. Archang, C. H. Kuan, W. M. Weaver, J. S. Weinstein, A. C. Feng, A. Ruccia, E. Sideris, V. Ragkousis, J. Koh, M. V. Plikus, D. Di Carlo, T. Segura, P. O. Scumpia, Nat. Mater. 2020, 20, 560.

[51] N. Asadi, H. Pazoki-Toroudi, A. R. Del Bakhshayesh, A. Akbarzadeh, S. Davaran, N. Annabi, Int. J. Biol. Macromol. 2020, 170, 728.

[52] J. Y. Li, D. J. Mooney, Nat. Rev. Mater. 2016, 1, 16071.

[53] K. Zhang, Y. Fang, Y. P. He, H. H. Yin, X. Guan, Y. Y. Pu, B. G. Zhou, W. W. Yue, W. W. Ren, D. Du, H. Y. Li, C. Liu, L. P. Sun, Y. Chen, H. X. Xu, Nat. Commun. 2019, 10, 5380.

[54] Y. F. Li, E. Kumacheva, Sci. Adv. 2018, 4, eaas8998.

[55] A. K. Mishra, T. J. Wallin, W. Y. Pan, P. Xu, K. Y. Wang, E. P. Giannelis, B. Mazzolai, R. F. Shepherd, Sci. Rob. 2020, 5, eaaz3918.

[56] X. Y. Liu, C. Steiger, S. T. Lin, G. A. Parada, J. Liu, H. F. Chan, H. Yuk, N. V. Phan, J. Collins, S. Tamang, G. Traverso, X. H. Zhao, Nat. Commun. 2019, 10, 493.

[57] J. Kim, A. S. Campbell, B. E. F. de Avila, J. Wang, Nat. Biotechnol. 2019, 37, 389.

[58] D. P. Browe, C. Wood, M. T. Sze, K. A. White, T. Scott, R. M. Olabisi, J. W. Freeman, Polymer 2017, 117, 331.

[59] X. F. Pan, Q. H. Wang, P. He, K. Liu, Y. H. Ni, L. H. Chen, X. H. Ouyang, L. L. Huang, H. P. Wang, S. Y. Xu, Chem. Eng. J. 2020, 379, 122271.

[60] Q. H. Wang, X. F. Pan, C. M. Lin, X. J. Ma, S. L. Cao, Y. H. Ni, Chem. Eng. J. 2020, 396, 125341.

[61] H. Liao, X. L. Guo, P. B. Wan, G. H. Yu, Adv. Funct. Mater. 2019, 29, 1904507.

[62] H. L. Sun, Y. Zhao, C. F. Wang, K. K. Zhou, C. Yan, G. Q. Zheng, J. J. Huang, K. Dai, C. T. Liu, C. Y. Shen, Nano Energy 2020, 76, 105035.

[63] J. Yang, X. B. Sun, Q. Kang, L. Zhu, G. Qin, Q. Chen, Polym. Test. 2021, 93, 106879. 
[64] W. H. Zheng, Y. Y. Li, L. J. Xu, Y. D. Huang, Z. X. Jiang, B. Li, Polymer 2020, 211, 123095

[65] F. Zhao, Y. Shi, L. J. Pan, G. H. Yu, Acc. Chem. Res. 2017, 50, 1734.

[66] Y. C. Chiu, S. Kocagoz, J. C. Larson, E. M. Brey, PLoS One 2013, 8, e60728.

[67] C. M. Ninciuleanu, R. Ianchis, E. Alexandrescu, C. I. Mihaescu, C. Scomoroscenco, C. L. Nistor, S. Preda, C. Petcu, M. Teodorescu, Materials 2021, 14, 2305.

[68] S. T. Lust, D. Hoogland, M. D. A. Norman, C. Kerins, J. Omar, G. M. Jowett, T. T. L. Yu, Z. Yan, J. Z. Xu, D. Marciano, R. M. P. da Silva, C. A. Dreiss, P. Lamata, R. J. Shipley, E. Gentleman, ACS Biomater. Sci. Eng. 2021, 7, 4293.

[69] J. P. Best, J. W. Cui, M. Mullner, F. Caruso, Langmuir 2013, 29, 9824.

[70] K. Xue, Z. P. Liu, Q. Y. Lin, J. Y. C. Lim, K. Y. Tang, S. L. Wong, B. H. Parikh, X. Y. Su, X. J. Loh, ACS Appl. Bio Mater. 2020, 3, 9043.

[71] P. Kunwar, A. V. S. Jannini, Z. Long, M. J. Ransbottom, J. S. Perkins, J. H. Henderson, J. M. Hasenwinkel, P. Soman, ACS Appl. Mater. Interfaces 2020, 12, 1640.

[72] E. M. Sanchez, J. C. Gomez-Blanco, E. L. Nieto, J. G. Casado, A. Macias-Garcia, M. A. D. Diez, J. P. Carrasco-Amador, D. T. Martin, F. M. Sanchez-Margallo, J. B. Pagador, Front. Bioeng. Biotech. 2020, 8, 776.

[73] D. Wang, D. F. E. Ker, K. W. Ng, K. Li, B. Gharaibeh, M. Safran, E. Cheung, P. Campbell, L. Weiss, Y. Z. P. Yang, NPG Asia Mater 2021, 13,26 .

[74] C. B. Goy, R. E. Chaile, R. E. Madrid, React. Funct. Polym. 2019, 145, 104314.

[75] J. N. Loos, C. E. Boott, D. W. Hayward, G. Hum, M. J. MacLachlan, Langmuir 2021, 37, 105.

[76] J. Raeburn, D. J. Adams, Chem. Commun. 2015, 51, 5170.

[77] W. Liyanage, K. Vats, A. Rajbhandary, D. S. W. Benoit, B. L. Nilsson, Chem. Commun. 2015, 51, 11260.

[78] S. Basu, R. Johl, S. Pacelli, S. Gehrke, A. Paul, ACS Macro Lett. 2020, 9, 1230.

[79] S. E. Gullbrand, T. P. Schaer, P. Agarwal, J. R. Bendigo, G. R. Dodge, W. Chen, D. M. Elliott, R. L. Mauck, N. R. Malhotra, L. J. Smith, Acta Biomater. 2017, 60, 201.

[80] R. Van Drunen, A. C. Jimenez-Vergara, E. H. Tsai, R. Tchen, T. Cagle, A. B. Agee, J. Roberts, J. M. Steele, D. J. Munoz-Pinto, ACS Appl. Bio Mater. 2019, 2, 975.

[81] F. Chen, P. Le, K. Lai, G. M. Fernandes-Cunha, D. Myung, Chem. Mater. 2020, 32, 5208.

[82] J. Yang, C. Gong, F. K. Shi, X. M. Xie, J. Phys. Chem. B 2012, 116, 12038

[83] Y. G. Wang, C. R. Garcia, Z. Y. Ding, R. Gabrilska, K. P. Rumbaugh, J. Y. Wu, Q. Y. Liu, W. Li, ACS Sustainable Chem. Eng. 2020, 8, 18006.

[84] K. H. Vining, A. Stafford, D. J. Mooney, Biomaterials 2019, 188, 187.

[85] J. Guo, C. Wei, X. Wang, Y. Hou, W. Guo, Int. J. Biol. Macromol. 2021, 180, 234.

[86] J. Lewandowska-Lancucka, A. Gilarska, A. Bula, W. Horak, A. Latkiewicz, M. Nowakowska, Int. J. Biol. Macromol. 2019, 136, 1196.

[87] K. E. Inostroza-Brito, E. C. Collin, A. Majkowska, S. Elsharkawy, A. Rice, A. E. D. Hernandez, X. Xiao, J. Rodriguez-Cabello, A. Mata, Acta Biomater. 2017, 58, 80.

[88] B. O. Okesola, A. Mata, Chem. Soc. Rev. 2018, 47, 3721.

[89] J. Z. Pan, Y. Jin, S. Q. Lai, L. J. Shi, W. H. Fan, Y. C. Shen, Chem. Eng. J. 2019, 370, 1228.

[90] X. Zhao, Soft Matter 2014, 10, 672

[91] L. J. Xu, C. Wang, Y. Cui, A. L. Li, Y. Qiao, D. Qiu, Sci. Adv. 2019, 5, eaau3442.

[92] S. Panpinit, S. A. Pongsomboon, T. Keawin, S. Saengsuwan, React. Funct. Polym. 2020, 156, 104739.

[93] S. Javanbakht, M. T. Nazeri, A. Shaabani, M. Ghorbani, Int. J. Biol. Macromol. 2020, 164, 2873.
[94] A. L. Chandgude, A. Domling, Green Chem. 2016, 18, 3718.

[95] J. Luo, G. S. Chen, S. J. Chen, Z. D. Li, Y. L. Liu, Chem. - Eur. J. 2021, 27, 6598.

[96] R. O. Rocha, M. O. Rodrigues, B. A. D. Neto, ACS Omega 2020, 5, 972.

[97] I. Ugi, C. Steinbruckner, Angew. Chem., Int. Ed. 1960, 72, 267.

[98] A. J. Kuijpers, G. H. Engbers, J. Krijgsveld, S. A. Zaat, J. Dankert, J. Feijen, J. Biomater. Sci., Polym. Ed. 2000, 11, 225.

[99] S. O. Sen, A. K. Nayak, P. Devbhuti, A. Ghosh, Int. J. Pharm. Pharm. Sci. 2019, 11, 1

[100] S. Maiti, P. S. Khillar, D. Mishra, N. A. Nambiraj, A. K. Jaiswal, Polym. Test. 2021, 97, 107155.

[101] B. Balakrishnan, A. Jayakrishnan, Biomaterials 2005, 26, 3941.

[102] M. M. Welz, C. M. Ofner, 3rd, J. Pharm. Sci. 1992, 81, 85

[103] T. Figueiredo, Y. Ogawa, J. Jing, V. Cosenza, I. Jeacomine, J. D. M. Olsson, T. Gerfaud, J. G. Boiteau, C. Harris, R. Auzely-Velty, Polym. Chem. 2020, 11, 3800 .

[104] R. Resmi, J. Parvathy, A. John, R. Joseph, Carbohyd. Polym. 2020, 234, 115902

[105] B. Balakrishnan, N. Joshi, A. Jayakrishnan, R. Banerjee, Acta Biomater. 2014, 10, 3650 .

[106] S. Turk, I. Altinsoy, G. C. Efe, M. Ipek, M. Ozacar, C. Bindal, Mater. Sci. Eng., C 2021, 121, 111829.

[107] H. Gao, C. Yu, Q. Li, X. Cao, Carbohydr. Polym. 2021, 258, 117663.

[108] X. C. Ding, Y. D. Wang, J. Mater. Chem. B 2017, 5, 887.

[109] B. Derkus, B. O. Okesola, D. W. Barrett, M. D'Este, T. T. Chowdhury, D. Eglin, A. Mata, Acta Biomater. 2020, 109, 82.

[110] M. K. Nguyen, C. T. Huynh, A. Gilewski, S. E. Wilner, K. E. Maier, N. Kwon, M. Levy, E. Alsberg, Sci. Adv. 2019, 5, eaax0801.

[111] C. Colquhoun, E. R. Draper, R. Schweins, M. Marcello, D. Vadukul, L. C. Serpell, D. J. Adams, Soft Matter 2017, 13, 1914.

[112] E. R. Draper, D. J. Adams, Chem 2017, 3, 390.

[113] J. K. Gupta, D. J. Adams, N. G. Berry, Chem. Sci. 2016, 7, 4713.

[114] L. A. Estroff, A. D. Hamilton, Chem. Rev. 2004, 104, 1201.

[115] J. A. Foster, J. W. Steed, Angew. Chem., Int. Ed. 2010, 49, 6718.

[116] F. Zhao, M. L. Ma, B. Xu, Chem. Soc. Rev. 2009, 38, 883.

[117] S. Panja, B. Dietrich, O. Shebanova, A. J. Smith, D. J. Adams, Angew. Chem., Int. Ed. 2021, 60, 9973.

[118] D. J. Cornwell, B. O. Okesola, D. K. Smith, Angew. Chem., Int. Ed. 2014, 53, 12461.

[119] K. J. Skilling, F. Citossi, T. D. Bradshaw, M. Ashford, B. Kellam, M. Marlow, Soft Matter 2014, 10, 237.

[120] C. C. Piras, P. Slavik, D. K. Smith, Angew. Chem., Int. Ed. 2020, 59, 853.

[121] H. Yuk, B. Lu, X. Zhao, Chem. Soc. Rev. 2019, 48, 1642.

[122] C. Christianson, N. N. Goldberg, D. D. Deheyn, S. Q. Cai, M. T. Tolley, Sci. Rob. 2018, 3, eaat 1893.

[123] X. Pu, M. M. Liu, X. Y. Chen, J. M. Sun, C. H. Du, Y. Zhang, J. Y. Zhai, W. G. Hu, Z. L. Wang, Sci. Adv. 2017, 3, e1700015.

[124] C. Lu, X. Chen, Acc. Chem. Res. 2020, 53, 1468.

[125] J. C. Yang, J. Mun, S. Y. Kwon, S. Park, Z. Bao, S. Park, Adv. Mater. 2019, 31, 1904765.

[126] S. Xia, Q. Zhang, S. X. Song, L. J. Duan, G. H. Gao, Chem. Mater. $2019,31,9522$.

[127] Z. X. Deng, Y. Guo, X. Zhao, P. X. Ma, B. L. Guo, Chem. Mater. 2018, $30,1729$.

[128] Y. Z. Zhang, K. H. Lee, D. H. Anjum, R. Sougrat, Q. Jiang, H. Kim, H. N. Alshareefit, Sci. Adv. 2018, 4, eaat0098.

[129] S. H. Hwang, Y. B. Park, S. H. Hur, H. G. Chae, ACS Appl. Nano Mater. 2018, 1, 2836.

[130] J. Wang, L. Y. Sun, M. H. Zou, W. Gao, C. H. Liu, L. R. Shang, Z. Z. Gu, Y. I. Zhao, Sci. Adv. 2017, 3, e1700004.

[131] X. Han, Z. Lv, F. Ran, L. Dai, C. Li, C. Si, Int. J. Biol. Macromol. 2021, $176,78$. 
[132] W. Y. Wang, K. Ouaras, A. L. Rutz, X. Li, M. Gerigk, T. E. Naegele, G. G. Malliaras, Y. Y. S. Huang, Sci. Adv. 2020, 6, eaba0931.

[133] P. He, R. S. Guo, K. Hu, K. Liu, S. Lin, H. Wu, L. L. Huang, L. H. Chen, Y. H. Ni, Chem. Eng. J. 2021, 414, 128726.

[134] Z. Y. Lei, P. Y. Wu, Nat. Commun. 2019, 10, 3429.

[135] Y. Zhang, M. Y. Li, X. Han, Z. W. Fan, H. H. Zhang, Q. L. Li, Chem. Phys. Lett. 2021, 769, 138437.

[136] A. Wang, Y. F. Wang, B. Zhang, K. N. Wan, J. X. Zhu, J. S. Xu, C. Zhang, T. X. Liu, Chem. Eng. J. 2021, 411, 128506.
[137] Y. Ko, J. Kim, H. Y. Jeong, G. Kwon, D. Kim, M. Ku, J. Yang, Y. Yamauchi, H. Y. Kim, C. Lee, J. You, Carbohydr. Polym. 2019, 203, 26.

[138] B. Lu, H. Yuk, S. Lin, N. Jian, K. Qu, J. Xu, X. Zhao, Nat. Commun. 2019, 10, 1043.

[139] T. Lim, Y. Kim, S. M. Jeong, C. H. Kim, S. M. Kim, S. Y. Park, M. H. Yoon, S. Ju, Sci. Rep. 2019, 9, 17294.

[140] A. Abedi, M. Hasanzadeh, L. Tayebi, Mater. Chem. Phys. 2019, 237, 121882.

[141] M. G. Azar, J. M. Dodda, P. Belsky, M. Slouf, V. Vavrunkova, J. Kadlec, T. Remis, Polym. Int. 2021.

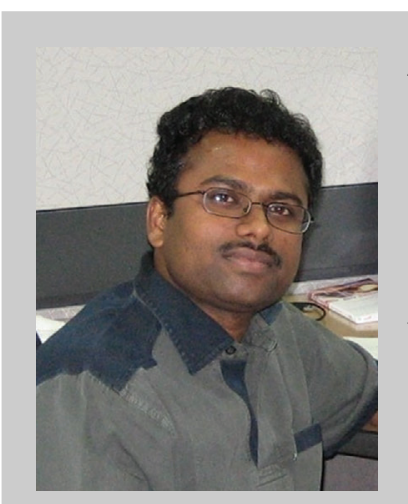

Jagan Mohan Dodda obtained his Ph.D. in Chemistry from the Central Salt \& Marine Chemicals Research Institute (Bhavnagar University), Gujarat, India. Later, he worked as a Postdoctoral researcher at Akita University (Japan), Korea Forest Research Institute (South Korea) and the University Sains Malaysia (Malaysia). Currently, he is employed as a Senior Researcher at the New Technology Research Institute, University of West Bohemia, Czech Republic. He specializes in the synthesis and characterization of composite membranes for organic/inorganic and bio-separations. His present research interest focuses on the development of tough and flexible hydrogels for various biomedical applications.

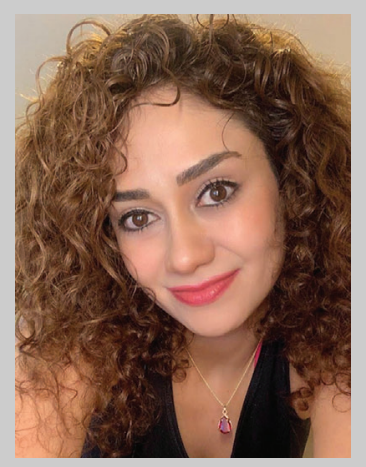

Mina Ghafouri Azar is a Ph.D. student who specializes in Organic Chemistry. She obtained her M.Sc. in Chemical Engineering from the Sejong University, South Korea in 2018. Later, she joined, the same University as a researcher. In 2019, she joined the New Technologies Research Centre, University of West Bohemia in Pilsen as a Ph.D. student. Presently, she is working on the synthesis of multicomponent hydrogels by using biocompatible polymers.

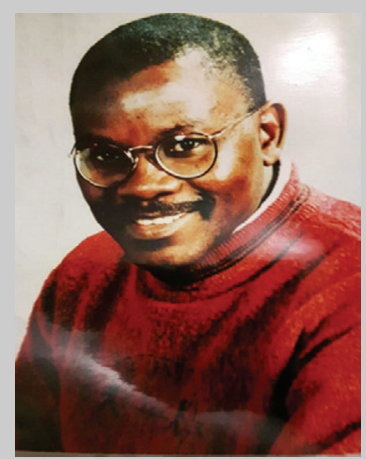

Emmanuel Rotimi Sadiku studied BSc. (Hons), 1980, and Ph.D., 1986, at the University of Strathclyde, Glasgow, Scotland, UK, where he specialized in Polymer Physics (Polymer Processing and X-ray Diffraction, Thermal Characteristics, Rheology and Microscopy of Polymers). He is currently (since January 2004), a Research Professor at the Tshwane University of Technology (TUT), Pretoria, RSA. This follows his several years of research exposures in different countries, following graduation: Italy, Sweden, Germany and University of Stellenbosch, RSA. This is in addition to his teaching experiences (a total of $15 \frac{1}{2}$ years), in two Nigerian Universities. 